\title{
Stability results on the circumference of a graph
}

\author{
Jie Ma* Bo Ning ${ }^{\dagger}$
}

\begin{abstract}
In this paper, we extend and refine previous Turán-type results on graphs with a given circumference. Let $W_{n, k, c}$ be the graph obtained from a clique $K_{c-k+1}$ by adding $n-(c-k+1)$ isolated vertices each joined to the same $k$ vertices of the clique, and let $f(n, k, c)=e\left(W_{n, k, c}\right)$. Improving a celebrated theorem of Erdös and Gallai [8, Kopylov [18] proved that for $c<n$, any 2-connected graph $G$ on $n$ vertices with circumference $c$ has at $\operatorname{most} \max \left\{f(n, 2, c), f\left(n,\left\lfloor\frac{c}{2}\right\rfloor, c\right)\right\}$ edges, with equality if and only if $G$ is isomorphic to $W_{n, 2, c}$ or $W_{n,\left\lfloor\frac{c}{2}\right\rfloor, c}$. Recently, Füredi et al. [15, 14] proved a stability version of Kopylov's theorem. Their main result states that if $G$ is a 2-connected graph on $n$ vertices with circumference $c$ such that $10 \leq c<n$ and $e(G)>\max \left\{f(n, 3, c), f\left(n,\left\lfloor\frac{c}{2}\right\rfloor-1, c\right)\right\}$, then either $G$ is a subgraph of $W_{n, 2, c}$ or $W_{n,\left\lfloor\frac{c}{2}\right\rfloor, c}$, or $c$ is odd and $G$ is a subgraph of a member of two well-characterized families which we define as $\mathcal{X}_{n, c}$ and $\mathcal{Y}_{n, c}$.

We prove that if $G$ is a 2-connected graph on $n$ vertices with minimum degree at least $k$ and circumference $c$ such that $10 \leq c<n$ and $e(G)>\max \{f(n, k+$ $\left.1, c), f\left(n,\left\lfloor\frac{c}{2}\right\rfloor-1, c\right)\right\}$, then one of the following holds:

(i) $G$ is a subgraph of $W_{n, k, c}$ or $W_{n,\left\lfloor\frac{c}{2}\right\rfloor, c}$,

(ii) $k=2, c$ is odd, and $G$ is a subgraph of a member of $\mathcal{X}_{n, c} \cup \mathcal{Y}_{n, c}$, or

(iii) $k \geq 3$ and $G$ is a subgraph of the union of a clique $K_{c-k+1}$ and some cliques $K_{k+1}$ 's, where any two cliques share the same two vertices.

This provides a unified generalization of the above result of Füredi et al. [15, 14] as well as a recent result of $\mathrm{Li}$ et al. [20] and independently, of Füredi et al. [12] on non-Hamiltonian graphs. A refinement and some variants of this result are also obtained. Moreover, we prove a stability result on a classical theorem of Bondy [2] on the circumference. We use a novel approach, which combines several proof ideas including a closure operation and an edge-switching technique. We will also discuss some potential applications of this approach for future research.
\end{abstract}

\section{Introduction}

All graphs in this paper are simple and finite. The circumference $c(G)$ of a graph $G$ is the length of a longest cycle in $G$. A graph $G$ is called Hamiltonian if $c(G)=|V(G)|$. Let $\delta(G)$ and $e(G)$ denote the minimum degree and the number of edges in $G$, respectively.

Determining the circumference of a graph is a classical problem in graph theory. It is well known that even determining if the graph is Hamiltonian is NP-hard. There has been

\footnotetext{
*School of Mathematical Sciences, University of Science and Technology of China, Hefei, Anhui 230026, China. Email: jiema@ustc.edu.cn. Research supported in part by National Natural Science Foundation of China grants 11501539 and 11622110 and Anhui Initiative in Quantum information Technologies grant AHY150200.

${ }^{\dagger}$ Center for Applied Mathematics, Tianjin University, Tianjin 300072, China. Email: bo.ning@tju.edu.cn. Research supported in part by National Natural Science Foundation of China grant 11601379 .
} 
extensive research investigating various relations between the circumference and other natural graph parameters. One such example is the famous theorem proved by Dirac [6] in 1952, which states that for any 2-connected graph $G, c(G) \geq \min \{2 \delta(G),|V(G)|\}$. In this paper, we mainly focus on the Turán-type problems on the circumference. One cornerstone in this direction is the following celebrated Erdős-Gallai theorem.

Theorem 1.1 (Erdős and Gallai [8]). For any graph $G$ on $n$ vertices, $\left.e(G) \leq \frac{c(G)(n-1)}{2}\right]$ This is sharp if $n-1$ is divisible by $c-1$ (where $c:=c(G)$ ), by considering the graph consisting of cliques $K_{c}$ 's sharing only one common vertex. Theorem 1.1 also implies that if an $n$-vertex graph $G$ contains no paths of length $k 2$ then $e(G) \leq \frac{(k-1) n}{2}$.

Bondy 2] generalized this theorem by showing the following.

Theorem 1.2 (Bondy [2]). Let $G$ be a graph on $n$ vertices and let $C$ be a longest cycle of $G$ of length $c$. Then the number of edges with at most one endpoint in $C$ is at most $\frac{c}{2} \cdot(n-c)$. In addition, if $G$ is 2-connected, then this number is at most $\left\lfloor\frac{c}{2}\right\rfloor \cdot(n-c)$.

Since there are at most $\left(\begin{array}{l}c \\ 2\end{array}\right)$ edges spanned in $V(C)$, we see that Theorem 1.2 indeed is a strengthening of Theorem $1.1,3$

Throughout this paper, let $W_{n, k, c}$ be the graph obtained from a clique $K_{c-k+1}$ by adding $n-(c-k+1)$ isolated vertices each joined to the same $k$ vertices of $K_{c-k+1}$, and

$$
f(n, k, c):=\left(\begin{array}{c}
c-k+1 \\
2
\end{array}\right)+k \cdot(n-c+k-1) .
$$

So $W_{n, k, c}$ has $n$ vertices, minimum degree $k$ and circumference $c$ with $e\left(W_{n, k, c}\right)=f(n, k, c)$.

\subsection{Stability on non-Hamiltonian graphs with large minimum degree}

For non-Hamiltonian graphs $G$ (that is, $c(G) \leq n-1$ ), Ore 21 proved that $e(G) \leq$ $\left(\begin{array}{c}n-1 \\ 2\end{array}\right)+1=f(n, 1, n-1)$. This was generalized further by Erdös [7].

Theorem 1.3 (Erdös [7]). If $G$ is a non-Hamiltonian graph on $n$ vertices with $\delta(G) \geq k$, where $1 \leq k \leq(n-1) / 2$, then $e(G) \leq \max \left\{f(n, k, n-1), f\left(n,\left\lfloor\frac{n-1}{2}\right\rfloor, n-1\right)\right\}$.

This bound is sharp for all $1 \leq k \leq(n-1) / 2$. Recently, Li and Ning [20], and independently, Füredi, Kostochka and Luo [12] proved a stability version of this theorem.

Theorem $1.4([20,12])$. Let $G$ be a non-Hamiltonian graph on $n$ vertices with $\delta(G) \geq k$, where $1 \leq k \leq(n-1) / 2$. If $e(G)>\max \left\{f(n, k+1, n-1), f\left(n,\left\lfloor\frac{n-1}{2}\right\rfloor, n-1\right)\right\}$, then $G$ is a subgraph of either $W_{n, k, n-1}$ or the edge-disjoint union of two cliques $K_{n-k}$ and $K_{k+1}$ sharing a common vertex.

Very recently, Füredi, Kostochka and Luo obtained a stronger stability theorem (and also some other related results) in [13].

\footnotetext{
${ }^{1}$ For a graph $G$ without cycles, we view $c(G)=2$.

${ }^{2}$ We specify that throughout this paper, a path of length $k$ has $k$ edges (and hence $k+1$ vertices).

${ }^{3}$ An improved version for 2-connected graphs can be found in Fan 9$]$.
} 


\subsection{Stability on graphs with given circumference}

There are many refinements of Theorem 1.1 in the literature, see [11, 19, 23, 18] or the survey [16]. Among them, Kopylov [18] proved the following strong version in 1977.

Theorem 1.5 (Kopylov [18]). Let $G$ be a 2-connected graph on $n$ vertices. If $c(G)=c \leq$ $n-1$, then $e(G) \leq \max \left\{f(n, 2, c), f\left(n,\left\lfloor\frac{c}{2}\right\rfloor, c\right)\right\}$.

We also mention that another proof of Theorem 1.5 was found by Fan, Lv, and Wang [10] in 2004. Using an edge-switching technique, the authors of [10] proved a slightly stronger result when $n-1 \geq c(G) \geq \frac{2 n}{3}+1$. This, together with a result of Woodall 23] that if $G$ is a 2-connected graph with circumference $c \leq \frac{2 n+2}{3}$ then $e(G) \leq f\left(n,\left\lfloor\frac{c}{2}\right\rfloor, c\right)$, gives a different proof of Theorem 1.5. More importantly for us, the technique of [10] provides an integral ingredient to the proof of our main theorem (see Subsection 4.3).

In 2016, Füredi, Kostochka, and Verstraëte [15] proved a stability result of Theorem 1.5 in the range of $n \geq 3\left\lfloor\frac{c}{2}\right\rfloor$. Together with this, Füredi, Kostochka, Luo, and Verstraëte 14] recently obtained a completed stability version of the above theorem of Kopylov. To state their result, we need to introduce two families $\mathcal{X}_{n, c}$ and $\mathcal{Y}_{n, c}$, which contain graphs of a given circumference $c$ where $c$ is odd, as follows:

- A graph $G$ in the family $\mathcal{X}_{n, c}$ has $n$ vertices and $V(G)=A \cup B \cup X$ such that $G[A]$ induces a clique $K_{\left\lfloor\frac{c}{2}\right\rfloor}$, both $G[B]$ and $G[X]$ are stable, $(A, B)$ is complete bipartite, and there exist two vertices $a \in A$ and $b \in B$ such that for any $x \in X, N_{G}(x)=\{a, b\}$.

- A graph $G$ in the family $\mathcal{Y}_{n, c}$ has $n$ vertices and $V(G)=A \cup B \cup Y$ such that $G[A]$ induces a clique $K_{\left\lfloor\frac{c}{2}\right\rfloor}, G[B]$ is stable, $G[Y]$ is a nontrivial star forest,$(A, B)$ is complete bipartite, and there exist two vertices $a, b \in A$ such that every star $S$ in $G[Y]$ is $\{a, b\}$ feasible: that is, $N_{G}(S)=\{a, b\}$ and if $|V(S)| \geq 3$, then all leaves of $S$ have degree 2 in $G$ and have a common neighbor in $\{a, b\}$.

Theorem 1.6 (Füredi, Kostochka, Luo, and Verstraëte [14]). Let G be a 2-connected graph on $n$ vertices with circumference $c$, where $10 \leq c \leq n-1$. If $e(G)>\max \left\{f(n, 3, c), f\left(n,\left\lfloor\frac{c}{2}\right\rfloor-\right.\right.$ $1, c)\}$, then one of the following conclusions holds:

(a) $G \subseteq W_{n, 2, c}$,

(b) $G \subseteq W_{n,\left\lfloor\frac{c}{2}\right\rfloor, c}$, or

(c) if $c$ is odd, then $G$ is a subgraph of a member of $\mathcal{X}_{n, c} \cup \mathcal{Y}_{n, c}$.

We remark that the case $c \leq 9$ was also fully characterized in [15, 14; in particular, the case $c=9$ requires another extremal graph, besides those stated in Theorem 1.6. As a corollary in [14, if in addition $G$ is 3-connected in Theorem [1.6, then one must have $G \subseteq W_{n,\left\lfloor\frac{c}{2}\right\rfloor, c}$.

By imposing minimum degree as a new parameter, Woodall [23] asked the following refinement of Theorem 1.1 in 1976.

Conjecture 1 (Woodall [23]). Let $G$ be a 2-connected graph on $n$ vertices with $\delta(G) \geq k$. If $c(G)=c \leq n-1$, then $e(G) \leq \max \left\{f(n, k, c), f\left(n,\left\lfloor\frac{c}{2}\right\rfloor, c\right)\right\}$.

One may also view this as a unification of Theorems 1.3 and 1.5. It should be mentioned that Kopylov's original proof in [18] can be modified to give a solution of this conjecture.

The Turán-type problem of cycles of given lengths for graphs with a given minimum degree is well-studied (see Chapter 5 of [1] for an inclusive discussion).

\footnotetext{
${ }^{4}$ We say a star forest is nontrivial, if it has at least two stars and every star has at least one edge.
} 


\subsection{The main result}

Our main result is a stability version of Woodall's conjecture, which also is a unified generalization of Theorem 1.6 and Theorem 1.4 for 2-connected graphs. We define the graph $Z_{n, k, c}$ to be the union of a clique $K_{c-k+1}$ and $\frac{n-(c-k+1)}{k-1}$ cliques $K_{k+1}$ 's such that any two cliques share the same two vertices.

Theorem 1.7. Let $G$ be a 2-connected graph on $n$ vertices with $\delta(G) \geq k$ and circumference $c$, where $10 \leq c \leq n-1$ If $e(G)>\max \left\{f(n, k+1, c), f\left(n,\left\lfloor\frac{c}{2}\right\rfloor-1, c\right)\right\}$, then one of the following conclusions holds:

(a) $G \subseteq W_{n, k, c}$,

(b) $G \subseteq W_{n,\left\lfloor\frac{c}{2}\right\rfloor, c}$,

(c) if $k=2$ and $c$ is odd, then $G$ is a subgraph of a member of $\mathcal{X}_{n, c} \cup \mathcal{Y}_{n, c}$, or

(d) if $k \geq 3$, then $G \subseteq Z_{n, k, c}$.

We make some remarks. First, we see that the case $k=2$ of Theorem 1.7 gives the precise statement of Theorem 1.6. Secondly, by letting $c=n-1$, Theorem 1.7 also provides a refined version of Theorem 1.4 for 2-connected graphs. Also we have $c \geq 2 k$ in Theorem 1.7, which follows by Dirac's theorem that $c \geq \min \{n, 2 k\}$. Note that $Z_{n, k, c}$ has $n$ vertices, minimum degree $k$ (assuming $c \geq 2 k$ ) and circumference $c$ with

$$
e\left(Z_{n, k, c}\right)=\left(\begin{array}{c}
c-k+1 \\
2
\end{array}\right)+\frac{k+2}{2} \cdot(n-c+k-1) .
$$

Thus in certain range it holds that $e\left(Z_{n, k, c}\right)>\max \left\{f(n, k+1, c), f\left(n,\left\lfloor\frac{c}{2}\right\rfloor-1, c\right)\right\}$.

We also notice that every graph in $\mathcal{X}_{n, c} \cup \mathcal{Y}_{n, c}$ has a vertex of degree 2, and the graph $Z_{n, k, c}$ has a 2-cut. Therefore, it is prompt to deduce that

Corollary 1.8. Let $G$ be a 3-connected graph on $n$ vertices with $\delta(G) \geq k$ and circumference $c$, where $10 \leq c \leq n-1$. If $e(G)>\max \left\{f(n, k+1, c), f\left(n,\left\lfloor\frac{c}{2}\right\rfloor-1, c\right)\right\}$, then either $G \subseteq W_{n, k, c}$ or $G \subseteq W_{n,\left\lfloor\frac{c}{2}\right\rfloor, c}$.

\subsection{A refinement}

Using a novel closure operation which we define below, we are able to refine Theorem 1.7 in more detail. We point out that the closure operation has proved to be a powerful tool for finding long cycles (see [3, 4, 22]). However it is surprising for us that in some cases one can even precisely describe the extremal graphs using closures.

The $k$-closure of a graph $G$ is the graph obtained from $G$ by recursively joining pairs of nonadjacent vertices whose degree sum is at least $k$ until no such pair remains. We also say that the resulting graph is $k$-closed. Let $G$ be a graph and $C$ be a cycle of $G$ of length c. The $C$-closure of $G$, denoted as $\bar{G}$, is obtained from $G$ by replacing the subgraph $G[C]$ by its $(c+1)$-closure. It is crucial to observe that $G \subseteq \bar{G}$.

Theorem 1.9. Let $G$ be a 2-connected graph on $n$ vertices with $\delta(G) \geq k$ and let $C$ be a longest cycle in $G$ of length $c \in[10, n-1]$. If $e(G)>\max \left\{f(n, k+1, c), f\left(n,\left\lfloor\frac{c}{2}\right\rfloor-1, c\right)\right\}$, then one of the following holds:

(a) $\bar{G}=W_{n, k, c}$, where $\bar{G}$ denotes the $C$-closure of $G$,

(b) $G \subseteq W_{n,\left\lfloor\frac{c}{2}\right\rfloor, c}$

(c) if $k=2$ and $c$ is odd, then $G$ is a subgraph of a member of $\mathcal{X}_{n, c} \cup \mathcal{Y}_{n, c}$, or

(d) if $k \geq 3$, then $\bar{G}=Z_{n, k, c}$.

\footnotetext{
${ }^{5}$ Following the proofs, we shall see that the same statement also holds for the case $c=8$.
} 


\subsection{Two variants}

The following two variants of the main result also can be obtained analogously, from which we see how the extremal graphs of Theorem 1.9 change as the parameters vary in the function $f(n, k, c)$.

Theorem 1.10. Let $G$ be a 2-connected graph on $n$ vertices with $\delta(G) \geq k$ and let $C$ be a longest cycle in $G$ of length $c \in[10, n-1]$. If $e(G)>\max \left\{f(n, k+1, c), f\left(n,\left\lfloor\frac{c}{2}\right\rfloor, c\right)\right\}$, then $\bar{G}=W_{n, k, c}$ or $Z_{n, k, c}$, where $\bar{G}$ denotes the $C$-closure of $G$.

Theorem 1.11. Let $G$ be a 2-connected graph on $n$ vertices with $\delta(G) \geq k$ and let $C$ be a longest cycle in $G$ of length $c \in[10, n-1]$. If $e(G)>\max \left\{f(n, k, c), f\left(n,\left\lfloor\frac{c}{2}\right\rfloor-1, c\right)\right\}$, then either $G \subseteq W_{n,\left\lfloor\frac{c}{2}\right\rfloor, c}$, or $k=2, c$ is odd and $G$ is a subgraph of a member of $\mathcal{X}_{n, c} \cup \mathcal{Y}_{n, c}$.

In particular, if we choose $c=n-1$ in Theorem 1.10, then it follows that $\bar{G}=W_{n, k, n-1}$. This is because that $Z_{n, k, n-1}$ is valid only for $k=2$, but when $k=2, W_{n, 2, c}$ and $Z_{n, 2, c}$ are identical. This provides another refined version of Theorem 1.4 for 2-connected graphs.

\subsection{Stability on a theorem of Bondy}

Our other result on the circumference of a graph is a stability version of Theorem 1.2 ,

Theorem 1.12. Let $G$ be a 2-connected graph on $n$ vertices and $C$ be a longest cycle in $G$ of length $c$, where $10 \leq c \leq n-1$. If the number of edges with at most one endpoint in $C$ is more than $\left(\left\lfloor\frac{c}{2}\right\rfloor-1\right)(n-c)$, then either $G \subseteq W_{n,\left\lfloor\frac{c}{2}\right\rfloor, c}$, or $c$ is odd and $G$ is a subgraph of a member of $\mathcal{X}_{n, c} \cup \mathcal{Y}_{n, c}$.

\subsection{Proof reduction}

In this subsection, we give a sketch of the proof of Theorem 1.9, which we emphasize is quite different from the existing ones in [12, 14, 15.

The proof will be split into two parts, according to the simple observation that given a longest cycle $C$ in the graph $G$ which has many edges, either the number of edges with at most one endpoint in $C$ is large or the number of edges spanned in $V(C)$ is large. The former case will be dealt with by Theorem 1.12, and the latter case will be handled by the following result.

Define $h(n, k):=\left(\begin{array}{c}n-k \\ 2\end{array}\right)+k(k-1)$. We point out that $h(n+1, k)=e\left(W_{n, k, n}\right)$.

Theorem 1.13. Let $G$ be a 2-connected graph on $n$ vertices with $\delta(G) \geq k$ and $C$ be a longest cycle in $G$ of length $c \in[6, n-1]$. If $e(G)>\max \left\{f(n, k+1, c), f\left(n,\left\lfloor\frac{c}{2}\right\rfloor-1, c\right)\right\}$ and $e(G[C])>h\left(c+1,\left\lfloor\frac{c}{2}\right\rfloor-1\right)$, then either $G \subseteq W_{n,\left\lfloor\frac{c}{2}\right\rfloor, c}$, or $\bar{G} \in\left\{W_{n, k, c}, Z_{n, k, c}\right\}$, where $\bar{G}$ denotes the $C$-closure of $G$.

We give the formal reduction of Theorem 1.9 to Theorems 1.12 and 1.13 .

Proof of Theorem 1.9. (Assuming Theorems 1.12 and 1.13.) Let $G, C$ be as in Theorem 1.9. We notice that $e(G)>f\left(n,\left\lfloor\frac{c}{2}\right\rfloor-1, c\right)=\left(\left\lfloor\frac{c}{2}\right\rfloor-1\right)(n-c)+h\left(c+1,\left\lfloor\frac{c}{2}\right\rfloor-1\right)$. So either $e(G-C)+e(G-C, C)>\left(\left\lfloor\frac{c}{2}\right\rfloor-1\right)(n-c)$ or $e(G[C])>h\left(c+1,\left\lfloor\frac{c}{2}\right\rfloor-1\right)$. If the former case occurs, then by Theorem 1.12, either $G \subseteq W_{n,\left\lfloor\frac{c}{2}\right\rfloor, c}$, or $c$ is odd and $G$ is a subgraph of a member of $\mathcal{X}_{n, c} \cup \mathcal{Y}_{n, c}$. As every graph in $\mathcal{X}_{n, c} \cup \mathcal{Y}_{n, c}$ has a vertex of degree 2 , it is only valid when $k=2$. So the latter case occurs. Then the assertion of Theorem 1.9 follows from Theorem 1.13. 


\subsection{Organization}

The rest of the paper is organized as follows. In Section 2, first we introduce notations and terminologies, which include an important concept 'locally maximal cycle' for our proofs; then we collect and prove some lemmas on cycles and closures. In Section 3, we prove Theorem 1.12. In Section 4, we prove a stronger version (Theorem 4.1) of Theorem 1.13, whose proof will be split into three technical lemmas. In Section 5, we complete the proofs of the two variants, i.e., Theorems 1.10 and 1.11, In Section 6, we conclude this paper by discussing some future research.

\section{Preliminaries}

\section{$2.1 \quad$ Notations}

Let $G$ be a graph and $H$ be a subgraph of $G$. We use $G-H$ to denote the resulting graph obtained from $G$ by deleting all vertices of $H$. If $H$ consists of only one vertex $v$, then we just write it as $G-v$. For convenience, sometime we would abuse the notation by using the subgraph $H$ as its vertex set. For instance, we often use $|H|$ to express $|V(H)|$. Let $A$ be a subset of $V(G)$. By $N_{H}(A)$, we mean the set of all vertices in $V(H) \backslash A$ which have at least one neighbor in $A$. We write $G[A]$ for the induced subgraph of $G$ on $A$. We say $A$ is stable, if $G[A]$ has no edges. If $H, H^{\prime}$ are two disjoint subgraphs (or subsets) in $G$, we define $\left(H, H^{\prime}\right)$ to be the induced bipartite subgraph of $G$ on the two parts $V(H)$ and $V\left(H^{\prime}\right)$. For $x, y \in V(G)$, an $(x, y)$-path is a path in $G$ with two endpoints $x$ and $y$, and an $(x, H, y)$-path is an $(x, y)$-path with all internal vertices in $V(H)$. We use $d_{H}^{*}(x, y)$ to denote the length of a longest $(x, H, y)$-path. We say $G$ is Hamiltonian-connected, if for any two vertices $x, y \in V(G)$, there exists an $(x, y)$-path which passes through every vertex in $G$. The clique number of $G$ is the maximum size of a clique in $G$. For a cycle or path $C$ with a given orientation, we denote $v^{+}$and $v^{-}$as the successor and predecessor of the vertex $v$ on $C$, respectively. For a subsect $A \subseteq V(C)$, by $A^{+}$(resp. $A^{-}$) we mean the set consisting of $v^{+}$(resp. $v^{-}$) for all $v \in A$. An $(x, y)$-path in $C$ sometime is also written as $C[x, y]$. Two edges are independent, if their endpoints are distinct.

Let $C$ be a cycle of a graph $G$ and $R$ be a component of $G-C$. A subset $M=$ $\left\{x_{1}, x_{2}, \ldots, x_{s}\right\}$ of $V(G)$ is called a strong attachment of $R$ to $C$, if $x_{i}$ 's lie on $C$ in a cyclic order, and for any ordered pair of vertices $x_{i}, x_{i+1}$, where $x_{s+1}=x_{1}$, there exist $y_{i}, y_{i+1} \in V(R)$ such that $x_{i} y_{i}, x_{i+1} y_{i+1}$ are independent edges.

A cycle $C$ is locally maximal in a graph $G$ if there is no cycle $C^{\prime}$ in $G$ such that $\left|E\left(C^{\prime}\right)\right|>|E(C)|$ and $\left|E\left(C^{\prime}\right) \cap E(C, G-C)\right| \leq 2$. This concept will play an important role in our proofs (for Section 4 especially). It seems that in most situations a locally maximal cycle $C$ captures the properties of a longest cycle, and yet it has its own advantages for counting the number of edges incident with $V(C)$.

Lastly, we consider the monotonicity of the function $f(n, k, c)$, where $n, c$ are fixed. Basic calculation shows that $f(n, k, c)=\frac{3}{2}\left[k^{2}-\left(\frac{4 c-2 n}{3}+1\right) k\right]+\frac{c^{2}+c}{2}$ is convex in $k$. So the maximum of $f(n, k, c)$ over an interval $[a, b]$ is always attained at either $k=a$ or $k=b$. Assuming that $10 \leq c \leq n-1$, we have $\left\lfloor\frac{c}{2}\right\rfloor-1 \geq \frac{c-1}{3}+\frac{1}{2} \geq \frac{2 c-n}{3}+\frac{1}{2}$, which implies that $f\left(n,\left\lfloor\frac{c}{2}\right\rfloor, c\right) \geq f\left(n,\left\lfloor\frac{c}{2}\right\rfloor-1, c\right)$. This inequality will be needed in the proof of Theorem 1.10 . 


\subsection{Some results on cycles}

We collect and prove some results on cycles here. The following result is due to Bondy [2, which strengthens Dirac's theorem.

Theorem 2.1 (Bondy [2]). Let $G$ be a 2-connected graph on $n$ vertices. If every vertex except for at most one vertex is of degree at least $k$, then $c(G) \geq \min \{n, 2 k\}$.

The next result, which was proved by Fan [9], can be viewed as an average-degree version of the classical Erdős-Gallai theorem. This will be frequently used in our coming proofs for finding long paths between some specified vertices.

Theorem 2.2 (Fan [9]). Let $x, y$ be two distinct vertices in a 2-connected graph G. Suppose that the average degree of the vertices other than $x$ and $y$ in $G$ is $r$, then the longest $(x, y)$-path in $G$ has length at least $r$, with equality if and only if $r$ is an integer and $G \in\{J, J-x y\}$, where $J$ denotes the union of some cliques $K_{r+1}$ which pairwise share the same vertices $x$ and $y$.

One can derive the following lemma from Theorem 2 of [9] (choosing $k=2$ ). 6

Lemma 2.3 (Theorem 2, 9]). Let $G$ be a 2-connected graph, $C$ be a longest cycle of length $c$ in $G$, and $H$ a component of $G-C$ which is 2-connected. If the average degree of the vertices of $H$ in $G$ is $r$, then $c \geq 2 r$, with equality only if $H$ is a clique $K_{r-1}$ in which every vertex has the same two neighbors on $C$.

The following lemma studies some properties of a strong attachment.

Lemma 2.4 (Lemma 1, 9]). Let $G$ be a graph, $C$ be a cycle in $G$, and $R$ a component of $G-C$. Let $T=\left\{u_{1}, u_{2}, \ldots, u_{t}\right\}$ be a maximum strong attachment of $R$ to $C, S=$ $N_{C}(R) \backslash T, t=|T|$ and $s=|S|$. Then,

(i) Every vertex in $S$ is joined to only one vertex in $R$.

(ii) For each $1 \leq i \leq t$, suppose that $N_{C}(R) \cap V\left(C\left[u_{i}, u_{i+1}\right]\right)=\left\{a_{0}, a_{1}, \ldots, a_{p}\right\}$, where $a_{0}=u_{i}, a_{p}=u_{i+1}$, and $a_{j}$ 's are in a cyclic order on $C$. Then there is a subscript $m$ such that $N_{R}\left(a_{j}\right)=N_{R}\left(a_{0}\right)$ for $0 \leq j \leq m$ and $N_{R}\left(a_{j}\right)=N_{R}\left(a_{p}\right)$ for $m+1 \leq j \leq p$.

(iii) If $C$ is a longest cycle in $G$ of length $c$ and $t \geq 2$, then $c \geq \sum_{i=1}^{t} d_{R}^{*}\left(u_{i}, u_{i+1}\right)+2 s$.

Lastly, we bound the clique number on a long cycle by some parameters related to a strong attachment.

Lemma 2.5. Let $G$ be a 2-connected graph, $C$ a locally maximal cycle in $G$, and $R$ a component of $G-C$. Let $T$ be a strong attachment of $R$ to $C$. Let $t=|T|, q=\left|N_{C}(R) \backslash T\right|$ and $\omega$ be the clique number of $G[C]$. If for any $x, x^{\prime} \in T$, the longest $\left(x, R, x^{\prime}\right)$-path is of length at least $d$, where $d \geq 2$, then the following hold:

(i) $\omega \leq|C|-(d-1)(t-1)$;

(ii) If $T$ is a maximum strong attachment, then $\omega \leq|C|-(d-1)(t-1)-q$.

Proof. We write $C=x_{1} x_{2} \ldots x_{c} x_{1}$ and view $x_{1}, x_{2}, \ldots, x_{c}$ appearing on $C$ in the clockwise order. (All subscripts are taken under modulo $c$ in this proof.) For $x, y \in V(C)$, by $C[x, y]$

\footnotetext{
${ }^{6}$ The original statement of Theorem 2 in 9 requires that " $C$ is locally longest with respect to $H$ and $H$ is locally 2-connected to $C$ ", which can be implied if $C$ is a longest cycle in $G$ and both $G$ and $H$ are 2 -connected as in Lemma 2.3
} 
we denote the segment of $C$ from $x$ to $y$ in the clockwise order. Let $T=\left\{u_{1}, u_{2}, \ldots, u_{t}\right\}$ and $u_{j}:=x_{i_{j}}$, where $1 \leq i_{1}<\ldots<i_{t} \leq c$. Let $W$ be a maximum clique in $G[C]$.

We first prove (i). Since $d_{R}^{*}\left(u_{j}, u_{j+1}\right) \geq d$ and $C$ is locally maximal, we see that $C\left[u_{j}, u_{j+1}\right]$ is a path of length at least $d$. For each $j$, let $A_{j}=V\left(C\left[x_{i_{j}+1}, x_{i_{j}+\left\lceil\frac{d-1}{2}\right\rceil}\right]\right)$ and $B_{j}=V\left(C\left[x_{i_{j+1}-\left\lfloor\frac{d-1}{2}\right\rfloor}, x_{i_{j+1}-1}\right]\right)$. So $A_{j}$ and $B_{j}$ are disjoint. Let $A=\cup_{j} A_{j}$ and $B=\cup_{k} B_{k}$. We claim that for any $j \neq k$, there are no edges between $A_{j}$ and $A_{k}$. Suppose this was not the case. Then there exist $1 \leq \ell, \ell^{\prime} \leq\left\lceil\frac{d-1}{2}\right\rceil$ such that $e:=x_{i_{j}+\ell} x_{i_{k}+\ell^{\prime}} \in E(G)$. Let $P$ be a $\left(u_{j}, R, u_{k}\right)$-path of length at least $d$. Then $C\left[x_{i_{k}+\ell^{\prime}}, x_{i_{j}}\right] \cup P \cup C\left[x_{i_{j}+\ell}, x_{i_{k}}\right] \cup\{e\}$ forms a cycle, say $C^{\prime}$. We see that $\left|C^{\prime}\right| \geq|C|+(d+1)-\left(\ell+\ell^{\prime}\right) \geq|C|+(d+1)-2\left\lceil\frac{d-1}{2}\right\rceil>|C|$ and $\left|E\left(C^{\prime}\right) \cap E(C, G-C)\right| \leq 2$, a contradiction to that $C$ is locally maximal, proving the claim. The claim shows that the maximum clique $W$ can intersect with at most one $A_{j}$, so $|V(W) \cap A| \leq\left\lceil\frac{d-1}{2}\right\rceil$. Similarly, there are no edges between $B_{j}$ and $B_{k}$ for any $j \neq k$, and thus $|V(W) \cap B| \leq\left\lfloor\frac{d-1}{2}\right\rfloor$. As $A$ and $B$ are disjoint, we have $|A \cup B|=(d-1) t$. Now we prove (i) by showing

$$
\begin{aligned}
\omega & =|V(W) \backslash(A \cup B)|+|V(W) \cap(A \cup B)| \leq|V(C) \backslash(A \cup B)|+|V(W) \cap(A \cup B)| \\
& \leq|V(C)|-|A \cup B|+|V(W) \cap A|+|V(W) \cap B| \leq c-(d-1)(t-1) .
\end{aligned}
$$

To prove (ii), we need a refined argument for (i). For any $1 \leq j \leq t$, let $N_{C}(R) \cap$ $V\left(C\left[u_{j}, u_{j+1}\right]\right)=\left\{a_{0}, a_{1}, \ldots, a_{p}\right\}$, where $a_{0}=u_{j}, a_{p}=u_{j+1}$, and $a_{\ell}$ 's for $0 \leq \ell \leq p$ appear on $C$ in the clockwise order. By Lemma 2.4. there is a subscript $m$ such that $N_{R}\left(a_{\ell}\right)=N_{R}\left(u_{j}\right)$ for $0 \leq \ell \leq m$ and $N_{R}\left(a_{\ell}\right)=N_{R}\left(u_{j+1}\right)$ for $m+1 \leq \ell \leq p$. Consider the segment $C_{j}:=C\left[a_{m}, a_{m+1}\right]$. Since $N_{R}\left(a_{m}\right)=N_{R}\left(u_{j}\right)$ and $N_{R}\left(a_{m+1}\right)=N_{R}\left(u_{j+1}\right)$, we see that $d_{R}^{*}\left(a_{m}, a_{m+1}\right)=d_{R}^{*}\left(u_{j}, u_{j+1}\right) \geq d$. So $C_{j}$ is a path of length at least $d$. Similarly as in the proof of (i), let $A_{j}$ be the set of the first $\left\lceil\frac{d-1}{2}\right\rceil$ vertices on $C_{j}$ (starting from $a_{m}$ but not including $a_{m}$ ), and let $B_{j}$ be the set of the last $\left\lfloor\frac{d-1}{2}\right\rfloor$ vertices on $C_{j}$ (not including $\left.a_{m+1}\right)$. Also let $A=\cup_{j} A_{j}$ and $B=\cup_{j} B_{j}$. So $A$ and $B$ are disjoint. Similarly, we can show that $W$ intersects with at most one $A_{j}$. Thus, $|V(W) \cap A| \leq\left\lceil\frac{d-1}{2}\right\rceil$, with equality if and only if $V(W) \cap A=A_{j}$ for some $j$. Also $|V(W) \cap B| \leq\left\lfloor\frac{d-1}{2}\right\rfloor$.

We consider $\left(N_{C}(R)\right)^{+}$. First we show $\left(N_{C}(R)\right)^{+}$is stable. Otherwise, there exist $x, y \in N_{C}(R)$ with $x^{+} y^{+} \in E(G)$; let $P$ be any $(x, R, y)$-path, which has length at least 2 , then $\left(C-\left\{x x^{+}, y y^{+}\right\}\right) \cup P \cup\left\{x^{+} y^{+}\right\}$is a cycle contradicting that $C$ is locally maximal. We point out that $\left(N_{C}(R)\right)^{+}$is disjoint from $B$, and it intersects with each $A_{j}$ in exactly one vertex $a_{m}^{+}$(i.e., the first vertex after $a_{m}$ in $\left.C_{j}\right)$. Let $D:=\left(N_{C}(R)\right)^{+} \backslash(A \cup B)$, where $|D|=\left|N_{C}(R)\right|-t=q$. We claim that $|V(W) \cap(A \cup D)| \leq\left\lceil\frac{d-1}{2}\right\rceil$. Since $D$ is stable, $W$ intersects with $D$ in at most one vertex. If $V(W) \cap D=\emptyset$, then this claim follows from that $|V(W) \cap A| \leq\left\lceil\frac{d-1}{2}\right\rceil$. So we may assume that $V(W) \cap D=\{x\}$ and $|V(W) \cap A|=\left\lceil\frac{d-1}{2}\right\rceil$. By the above analysis, we then have $V(W) \cap A=A_{j}$ for some $j$. In particular, the vertex $a_{m}^{+} \in A_{j}$ is in $W$. But then there are two vertices $x, a_{m}^{+}$in $V(W) \cap\left(N_{C}(R)\right)^{+}$, a contradiction. This proves the claim. Combining the above bounds, we have

$$
\begin{aligned}
\omega & \leq|V(C) \backslash(A \cup B \cup D)|+|V(W) \cap(A \cup B \cup D)| \\
& \leq|V(C)|-|A \cup B \cup D|+(d-1) \leq c-(d-1)(t-1)-q .
\end{aligned}
$$

This finishes the proof of Lemma 2.5.

\subsection{Lemmas on closures}

In this subsection, we prove some lemmas on $C$-closures. 
Lemma 2.6. Let $G$ be a graph on $n$ vertices. Then for any $x, y \in V(G)$, the longest $(x, y)$-path in the $(n+1)$-closure of $G$ has the same length as the longest $(x, y)$-path in $G$.

Proof. It suffices to prove the following: for two nonadjacent vertices $u, v$ in $G$ with $d_{G}(u)+$ $d_{G}(v) \geq n+1$ and for any $x, y \in V(G)$, there exists a longest $(x, y)$-path $P$ in $G^{\prime}:=G+\{u v\}$ satisfying that $E(P) \subseteq E(G)$. Suppose this is not true. Then any longest $(x, y)$-path $P$ in $G^{\prime}$ must contain the new edge $u v$. Assume that $x, u, v, y$ lie on $P$ in this order. First we observe that there is no common neighbor of $u$ and $v$ in $V(G)-V(P)$, as otherwise one can find an $(x, y)$-path longer than $P$ in $G^{\prime}$. Let $P_{1}:=P[x, u]$ and $P_{2}:=P[v, y]$. We claim that there are no vertices $a \in N_{G}(u) \cap V\left(P_{1}\right)$ and $b \in N_{G}(v) \cap V\left(P_{1}\right)$ such that $b=a^{+}$(we view $P$ from $x$ to $y$ ). Suppose such $a, b$ exist. Then $b \in V\left(P_{1}\right) \backslash\{u\}$. By Posa's rotation technique, $(P-\{a b, u v\}) \cup\{a u, b v\}$ is a longest $(x, y)$-path in $G^{\prime}$, however all its edges are from $E(G)$, a contradiction. This shows that $\left(N_{G}(u) \cap V\left(P_{1}\right)\right)^{+} \cap$ $\left(N_{G}(v) \cap V\left(P_{1}\right)\right)=\emptyset$. So $\left|N_{G}(u) \cap V\left(P_{1}\right)\right|+\left|N_{G}(v) \cap V\left(P_{1}\right)\right| \leq\left|V\left(P_{1}\right)\right|$. Similarly, we have $\left|N_{G}(u) \cap V\left(P_{2}\right)\right|+\left|N_{G}(v) \cap V\left(P_{2}\right)\right| \leq\left|V\left(P_{2}\right)\right|$. Combining the above bounds, it follows that $d_{G}(u)+d_{G}(v) \leq|V(G)|=n$, contradicting that $d_{G}(u)+d_{G}(v) \geq n+1$. This proves the lemma.

Lemma 2.7. Let $G$ be a graph and $C$ be a locally maximal cycle of $G$. Then $C$ is also a locally maximal cycle of the $C$-closure of $G$.

Proof. Let $\bar{G}$ denote the $C$-closure of $G$. We point out that $\bar{G}-C=G-C$ and $E(C, \bar{G}-$ $C)=E(C, G-C)$. Suppose this is not true. Then there is a cycle $D$ in $\bar{G}$ such that $|D|>|C|$ and $|E(D) \cap E(C, \bar{G}-C)| \leq 2$; and subject to this, we choose $D$ such that $|D|$ is maximum. It is fair to assume that $|E(D) \cap E(C, \bar{G}-C)|=2$ (as otherwise $E(D) \cap E(C, \bar{G}-C)=\emptyset$, implying that $D \subseteq G-C)$. Let $x y, x^{\prime} y^{\prime}$ be the two edges in the intersection, where $x, x^{\prime} \in V(C)$. Then $D$ consists of two internally disjoint $\left(x, x^{\prime}\right)$-paths $P_{1}$ and $P_{2}$, where $P_{1}$ is an $\left(x, G-C, x^{\prime}\right)$-path and $P_{2}$ is an $\left(x, x^{\prime}\right)$-path in $\bar{G}[C]$. Note that $P_{1}$ is in $G$, and by the maximality of $D, P_{2}$ is a longest $\left(x, x^{\prime}\right)$-path in $\bar{G}[C]$. By Lemma 2.6. there exists an $\left(x, x^{\prime}\right)$-path $P_{3}$ in $G[C]$ with $\left|P_{2}\right|=\left|P_{3}\right|$. Set $C^{\prime}:=P_{1} \cup P_{3}$. Then $C^{\prime}$ is a cycle in $G$ of length $\left|C^{\prime}\right|=\left|P_{1}\right|+\left|P_{3}\right|=\left|P_{1}\right|+\left|P_{2}\right|=|D|>|C|$. Furthermore, $\left|E\left(C^{\prime}\right) \cap E(C, G-C)\right|=|E(D) \cap E(C, \bar{G}-C)| \leq 2$, which contradicts the fact that $C$ is locally maximal in $G$. This completes the proof.

Lemma 2.8. Let $G$ be a 2-connected graph on $n$ vertices and $C$ be a locally maximal cycle in $G$ of length $c$, where $c \leq n-1$. Let $\bar{G}$ denote the $C$-closure of $G$. Then $\bar{G}[C]$ is non-Hamiltonian-connected.

Proof. Suppose for a contradiction that $\bar{G}[C]$ is Hamiltonian-connected. As $c \leq n-1$, there is a component $R$ in $G-C$. Since $G$ is 2-connected, there exist two distinct vertices $x, x^{\prime} \in N_{C}(R)$. Let $P_{1}$ be an $\left(x, R, x^{\prime}\right)$-path. Since $\bar{G}[C]$ is Hamiltonian-connected, there is an $\left(x, x^{\prime}\right)$-path $P_{2}$ in $\bar{G}[C]$, which passes through all vertices in $V(C)$. Then $C^{\prime}:=P_{1} \cup P_{2}$ is a cycle in $\bar{G}$ which is longer than $C$ and $\left|E\left(C^{\prime}\right) \cap E(C, \bar{G}-C)\right| \leq 2$. This contradicts Lemma 2.7 that $C$ is locally maximal in $\bar{G}$. This proves the lemma.

We need a theorem of Chvátal [5] on the degree sequences of non-Hamiltonian graphs.

Theorem 2.9 (Chvátal [5]). Let $G$ be a graph with degree sequence $d_{1} \leq d_{2} \leq \ldots \leq d_{n}$ and $n \geq 3$. If $G$ is non-Hamiltonian, then there is some integer $s<n / 2$ such that $d_{s} \leq s$ and $d_{n-s}<n-s$. 
We can get a corollary of Chvátal's theorem on non-Hamiltonian-connected graphs.

Lemma 2.10. Let $G$ be a non-Hamiltonian-connected graph on $n$ vertices with minimum degree at least 2. Then there exists a set of $s-1$ vertices in $G$ of degree at most $s$, where $2 \leq s \leq\left\lfloor\frac{n}{2}\right\rfloor$.

Proof. Since $G$ is non-Hamiltonian-connected, there exist $x, y \in V(G)$ such that there is no Hamiltonian path from $x$ to $y$ in $G$. Let $G^{\prime}$ be obtained from $G$ by adding a new vertex $z$ and two edges $x z, y z$. Clearly, $G^{\prime}$ is not Hamiltonian. Let $d_{1} \leq d_{2} \leq \ldots \leq d_{n+1}$ be the degree sequence of $G^{\prime}$. Since $\delta(G) \geq 2$, we have $d_{1}=2$, which denotes the degree of $z$ in $G^{\prime}$. By Theorem 2.9, there is some integer $s<\frac{n+1}{2}$ such that $d_{s} \leq s$ and $d_{n+1-s}<n+1-s$. As $d_{1}=2$, we see that $2 \leq s \leq\left\lfloor\frac{n}{2}\right\rfloor$. If we let $f_{1} \leq f_{2} \leq \ldots \leq f_{n}$ be the degree sequence of $G$, then each $f_{i}$ corresponds to the vertex associated with $d_{i+1}$ and thus $f_{i} \leq d_{i+1}$. This shows that $f_{s-1} \leq d_{s} \leq s$, proving the lemma.

The next lemma (in particular, its special case when $\delta=1$ ) will play an important role in the proof of Theorem 4.1. We establish a general version for possible studies in future. Its proof is analogous to Lemma 6 in [12].

Lemma 2.11. Let $G_{c}$ be a graph on c vertices with minimum degree at least 2. Further suppose that $G_{c}$ is $(c+1)$-closed and non-Hamiltonian-connected with

$$
e\left(G_{c}\right)>h\left(c+1,\left\lfloor\frac{c}{2}\right\rfloor-p\right) \text { for some integer } p \geq 0 .
$$

Then one of the following holds:

(i) $G_{c}$ contains a subset $S$ of $s-1$ vertices of degree at most $s$, where $2 \leq s \leq\left\lfloor\frac{c}{2}\right\rfloor-p-1$, such that $G_{c}-S$ is a clique; or

(ii) $G_{c}$ contains a subset $T$ of $t-1$ vertices of degree at most $t$, where $\left\lfloor\frac{c}{2}\right\rfloor-p+1 \leq t \leq\left\lfloor\frac{c}{2}\right\rfloor$.

Proof. Suppose neither (i) nor (ii) holds. Since $G_{c}$ is non-Hamiltonian-connected, by Lemma 2.10, there exists some $2 \leq s \leq\left\lfloor\frac{c}{2}\right\rfloor$ such that $G_{c}$ contains $s-1$ vertices of degree at most $s$. Subject to this, we choose $s$ to be maximal, and let $S$ be the set of all vertices in $G_{c}$ with degree at most $s$. If $\left\lfloor\frac{c}{2}\right\rfloor-p+1 \leq s \leq\left\lfloor\frac{c}{2}\right\rfloor$, then (ii) holds. If $s=\left\lfloor\frac{c}{2}\right\rfloor-p$, then $e\left(G_{c}\right) \leq(s-1) s+\left(\begin{array}{c}c-s+1 \\ 2\end{array}\right)=h(c+1, s)=h\left(c+1,\left\lfloor\frac{c}{2}\right\rfloor-p\right)$, a contradiction. So we may assume that $2 \leq s \leq\left\lfloor\frac{c}{2}\right\rfloor-p-1$. Moreover, by the maximality of $s$, we have $|S|=s-1$.

Next, we will show that $G_{c}-S$ is a clique. Suppose that there are nonadjacent vertices $u, v \in V\left(G_{c}\right)-S$. Without loss of generality, assume that $u$ is the one with the maximal degree among all vertices in $V\left(G_{c}\right)-S$, each of which is not adjacent to every vertex in $V\left(G_{c}\right)-S$. Let $S^{\prime}:=V(G)-N(u)-\{u\}$ and $s^{\prime}:=\left|S^{\prime}\right|+1=c-d(u)$. For any $w \in S^{\prime}$, since $w u \notin E\left(G_{c}\right)$ and $G_{c}$ is $(c+1)$-closed, we have $d(w) \leq c-d(u)=s^{\prime}$. So $S^{\prime}$ is a set of $s^{\prime}-1$ vertices of degree at most $s^{\prime}$. Since $v \notin S$, by the maximality of $S$, it follows that $d(v)>s$. So $s<d(v) \leq s^{\prime}$. By the maximality of $s$, we get that $s^{\prime} \geq\left\lfloor\frac{c}{2}\right\rfloor+1$. As $s^{\prime}=c-d(u)$, we get $d(u) \leq\left\lfloor\frac{c}{2}\right\rfloor$. We then claim that any vertex $x \in S^{\prime}$ has degree at most $\left\lfloor\frac{c}{2}\right\rfloor$. Indeed, if $x \in S$ then $d(x) \leq s \leq\left\lfloor\frac{c}{2}\right\rfloor-p-1$; otherwise $x \in S^{\prime} \backslash S$, then by the choice of $u, d(x) \leq d(u) \leq\left\lfloor\frac{c}{2}\right\rfloor$. Now observe that $S^{\prime}$ is a set of at least $\left\lfloor\frac{c}{2}\right\rfloor$ vertices of degree at most $\left\lfloor\frac{c}{2}\right\rfloor$, so $(i i)$ holds, a contradiction. This shows that $G_{c}-S$ is a clique and thus $(i)$ holds. This proves the lemma.

We remark that if $p=0$, then only (i) occurs in Lemma 2.11. 


\section{Stability on a theorem of Bondy}

In this section, we prove a stability result on a classic theorem due to Bondy [2]. We restate the statement here for the convenience of the readers.

Theorem 1.12. Let $G$ be a 2-connected graph on $n$ vertices and $C$ be a longest cycle in $G$ of length $c$, where $10 \leq c \leq n-1$. If $e(G-C)+e(G-C, C)>\left(\left\lfloor\frac{c}{2}\right\rfloor-1\right)(n-c)$, then either $G \subseteq W_{n,\left\lfloor\frac{c}{2}\right\rfloor, c}$ or $c$ is odd and $G$ is a subgraph of a member of $\mathcal{X}_{n, c} \cup \mathcal{Y}_{n, c}$.

To prove Theorem 1.12, a crucial step is to find a vertex in $G-C$ with $\left\lfloor\frac{c}{2}\right\rfloor$ neighbors in $C$ (see Theorem 3.1); this will be done in Subsection 3.1. As we shall see later (somehow surprisingly), the existence of such a vertex can give a lot of structural information of the graph $G$. We then complete the proof of Theorem 1.12 in Subsection 3.2 .

\subsection{A vertex with large degree}

In this subsection, we prove the following result.

Theorem 3.1. Let $G$ be a 2-connected graph on $n$ vertices and $C$ be a longest cycle in $G$ of length $c$, where $10 \leq c \leq n-1$. If $e(G-C)+e(G-C, C)>\left(\left\lfloor\frac{c}{2}\right\rfloor-1\right)(n-c)$, then there exists an isolated vertex $u$ in $G-C$ with $d_{C}(u)=\left\lfloor\frac{c}{2}\right\rfloor$.

Just as in the original theorem of Bondy, we also can drop off the connectivity condition. A more general statement is as follows.

Theorem 3.2. Let $G$ be a graph on $n$ vertices and $C$ be a longest cycle in $G$ of length $c$, where $4 \leq c \leq n-1$. If $e(G-C)+e(G-C, C)>\left(\left\lfloor\frac{c}{2}\right\rfloor-1\right)(n-c)$, then one of the following holds:

(a) $c \in\{6,7,9\}$;

(b) there exists a vertex $u \in V(G-C)$ with $d_{C}(u)=\left\lfloor\frac{c}{2}\right\rfloor$;

(c) there exists a cycle $C^{\prime}$ in $G$ satisfying that $\left|V\left(C \cap C^{\prime}\right)\right| \leq 1$ and

- if $V\left(C \cap C^{\prime}\right)=\emptyset$, then $\left|C^{\prime}\right| \geq 2\left\lfloor\frac{c}{2}\right\rfloor-3$,

- if $\left|V\left(C \cap C^{\prime}\right)\right|=1$, then $\left|C^{\prime}\right| \geq 2\left\lfloor\frac{c}{2}\right\rfloor-1$.

Proof. We prove the theorem by contradiction. Suppose that there exists an $n$-vertex non-Hamiltonian graph $G$ and a longest cycle $C$ in $G$ of length $c \geq 4$ such that $e(G-$ $C)+e(G-C, C)>\left(\left\lfloor\frac{c}{2}\right\rfloor-1\right)(n-c)$ and none of $(a),(b)$ and $(c)$ holds. We choose such a counterexample $G$ that $c$ is minimum and subject to this, the order $n$ is minimum. Throughout this proof, let $H:=G-C$ and so

$$
e(H)+e(H, C)>\left(\left\lfloor\frac{c}{2}\right\rfloor-1\right)(n-c) .
$$

Claim 1. $c \geq 5$ and $n \geq c+2$.

Proof. Assume that $c=4$. Then by (11) we have $e(H)+e(H, C)>n-4$. Suppose that there is a cycle $C^{\prime}$ in $G-E(C)$. So $\left|C^{\prime}\right|=3$ or 4 . If $\left|V\left(C^{\prime} \cap C\right)\right| \leq 1$, then clearly (c) holds; otherwise $\left|V\left(C^{\prime} \cap C\right)\right| \geq 2$, then there exists either a cycle longer than $C$ or a vertex in $H$ with two neighbors in $C$ (thus (b) holds), a contradiction. So there is no cycle in $G-E(C)$. Consider any component $R$ in $H$, which must be a tree. If $d_{C}(R) \geq 2$, then either there is a vertex in $R$ with two neighbors in $C$, or we can find a longer cycle, a contradiction. Thus $d_{C}(R) \leq 1$ and as $G-E(C)$ has no cycles, $e(R, C) \leq 1$. 
This implies that $e(R)+e(R, C) \leq|R|$. Summing over all components $R$ in $H$, we have $e(H)+e(H, C) \leq \sum|R|=n-4$, a contradiction. This proves that $c \geq 5$.

Now suppose that $n=c+1$. Let $V(H)=\{u\}$. By (11), $d_{C}(u) \geq\left\lfloor\frac{c}{2}\right\rfloor$. Since $C$ is a longest cycle in $G$, we must have $d_{C}(u)=\left\lfloor\frac{c}{2}\right\rfloor$. This proves Claim 1.

Claim 2. For any vertex $v \in V(H), d_{G}(v) \geq\left\lfloor\frac{c}{2}\right\rfloor$.

Proof. Suppose for a contradiction that there exists a vertex $u \in V(H)$ with $d_{G}(u) \leq$ $\left\lfloor\frac{c}{2}\right\rfloor-1$. Set $G^{\prime}:=G-u$. So $C$ remains a longest cycle in $G^{\prime}$. By Claim $1, n \geq c+2$, implying that $G^{\prime}$ is non-Hamiltonian. We also have $e\left(G^{\prime}-C\right)+e\left(G^{\prime}-C, C\right)=e(H)+$ $e(H, C)-d_{G}(u)>\left(\left\lfloor\frac{c}{2}\right\rfloor-1\right)(n-c-1)$. By the choice of $G$, one of $(a),(b)$ and $(c)$ holds in $G^{\prime}$. It is obvious to see that the same case also holds in $G$. This proves the claim.

Claim 3. $H$ is connected.

Proof. Suppose that $H$ is not connected. Then by averaging, there exists a component $R$ in $H$ such that $e(G[R])+e(G[R], C)>\left(\left\lfloor\frac{c}{2}\right\rfloor-1\right) \cdot|R|$. It is clear that $G[R \cup C]$ is non-Hamiltonian. Then by the choice of $G$, one of $(a),(b)$ and $(c)$ holds in $G[R \cup C]$, which also holds in $G$, a contradiction. This proves Claim 3.

Claim 4. $G$ is 2-connected.

Proof. Suppose that $G$ is not 2-connected. Then there exists an end-block 8 of $G$ such that $|V(B \cap C)| \leq 1$. Let $b$ be the unique cut-vertex of $G$ with $b \in V(B)$ (if it exists). By Claim 2, every vertex in $V(B)$, except the vertex $b$, has degree at least $\left\lfloor\frac{c}{2}\right\rfloor$ in $B$. By Theorem 2.1, we have $c(B) \geq \min \left\{|B|, 2\left\lfloor\frac{c}{2}\right\rfloor\right\}$. If $|B| \geq 2\left\lfloor\frac{c}{2}\right\rfloor-1$, then $c(B) \geq 2\left\lfloor\frac{c}{2}\right\rfloor-1$, a contradiction to $(c)$. Hence we may assume that $|B| \leq 2\left\lfloor\frac{c}{2}\right\rfloor-2$.

Let $H_{1}:=G-(B-b)$. Clearly $C$ is still a longest cycle in $H_{1}$. We claim that $H_{1}$ is not Hamiltonian. Indeed, otherwise $C$ must be a Hamiltonian cycle of $H_{1}$ and thus we have $H=B-b$. So $\frac{(|B|-1)|B|}{2} \geq e(B)=e(H)+e(H, C)>\left(\left\lfloor\frac{c}{2}\right\rfloor-1\right)(n-c)=\left(\left\lfloor\frac{c}{2}\right\rfloor-1\right)(|B|-1)$, which implies that $|B| \geq 2\left\lfloor\frac{c}{2}\right\rfloor-1$, a contradiction.

Note that we have $e\left(H_{1}\right)=e(G)-e(B)$. So $e\left(H_{1}-C\right)+e\left(H_{1}-C, C\right)=e\left(H_{1}\right)-$ $e(G[C])=e(G-C)+e(G-C, C)-e(B)>\left(\left\lfloor\frac{c}{2}\right\rfloor-1\right)(n-c)-\frac{|B|(|B|-1)}{2}$. Since $|B| \leq 2\left\lfloor\frac{c}{2}\right\rfloor-2$, it follows that $e\left(H_{1}-C\right)+e\left(H_{1}-C, C\right)>\left(\left\lfloor\frac{c}{2}\right\rfloor-1\right)\left(\left|V\left(H_{1}\right)\right|-c\right)$. By the choice of $G$, one of $(a),(b)$ and $(c)$ holds in $H_{1}$, which also holds in $G$. This proves Claim 4.

Claim 5. $|V(H)| \geq 3$.

Proof. Otherwise, in view of Claims 1 and 3, we may assume that $H$ is just an edge $v_{1} v_{2}$. So we have $e(H, C) \geq 2\left(\left\lfloor\frac{c}{2}\right\rfloor-1\right)$. Let $T=\left\{u_{1}, u_{2}, \ldots, u_{t}\right\} \subset N_{C}(H)$ be a maximum strong attachment of $H$ to $C$. Let $S:=N_{C}(H) \backslash T, t=|T|$ and $s=|S|$. For any $u_{i}, u_{i+1} \in T$, the $\left(u_{i}, H, u_{i+1}\right)$-path is of at least length 3. By Lemma 2.4, we have $e(H, C) \leq 2 t+s$ and $c \geq 3 t+2 s \geq \frac{3}{2} e(H, C) \geq 3\left(\left\lfloor\frac{c}{2}\right\rfloor-1\right)$. From this, we can derive a contradiction if $c \geq 8$ is even or $c \geq 11$ is odd. Thus, $c \in\{5,6,7,9\}$. It only needs to consider $c=5$, as otherwise $(a)$ holds. In case of $c=5$, we have $e(H, C) \geq 2$ and as $G$ is 2-connected, there are two independent edges in $(H, C)$, which would lead to a cycle of length at least 6 , a contradiction. This proves Claim 5 .

\footnotetext{
${ }^{7}$ A block $B$ in a graph $G$ is a maximal connected subgraph of $G$ such that there exists no cut-vertex of $B$. An end-block in $G$ is a block in $G$ containing at most one cut-vertex of $G$.
} 
Claim 6. $H$ is 2-connected.

Proof. Suppose that $H$ is not 2-connected. As $|V(H)| \geq 3$, there exist two end-blocks $B_{1}, B_{2}$ of $H$. Let $b_{i}$ be the unique cut-vertex of $H$ with $b_{i} \in V\left(B_{i}\right)$ for $i \in\{1,2\}$. Since $G$ is 2-connected, there exists a vertex $v_{2} \in V\left(B_{2}-b_{2}\right)$ with a neighbor $u_{2} \in V(C)$.

First assume that $B_{1}$ is an edge, say $v_{1} b_{1}$. By Claim 2, we have $d_{C}\left(v_{1}\right) \geq\left\lfloor\frac{c}{2}\right\rfloor-1$. If $d_{C}\left(v_{1}\right) \geq\left\lfloor\frac{c}{2}\right\rfloor$, then $(b)$ holds, a contradiction. Thus, $d_{C}\left(v_{1}\right)=\left\lfloor\frac{c}{2}\right\rfloor-1$. Notice that there is a $\left(v_{1}, v_{2}\right)$-path in $H$ of length at least 2. If $u_{2}$ is the unique neighbor of $v_{1}$ on $C$, then we have $c=5$ and there exists a cycle in $G\left[H \cup\left\{u_{2}\right\}\right]$ of length at least 4 , a contradiction to $(c)$. Hence, we may assume that $N_{C}\left(v_{1}\right) \backslash\left\{u_{2}\right\} \neq \emptyset$. Let $w_{1}, w_{2}, \ldots, w_{t}$ be the neighbors of $v_{1}$ on $C$ which appear in a cyclic order, where $t=d_{C}\left(v_{1}\right)=\left\lfloor\frac{c}{2}\right\rfloor-1$. For any $w_{i} \in N_{C}\left(v_{1}\right) \backslash\left\{u_{2}\right\}$, since there exists a $\left(w_{i}, H, u_{2}\right)$-path of length at least 4 , any $\left(w_{i}, u_{2}\right)$-segment of $C$ has length at least 4 . So we have $c \geq 8$ and thus $t \geq 3$. Let $w_{i}, w_{j} \in N_{C}\left(v_{1}\right) \backslash\left\{u_{2}\right\}$ be two vertices such that $u_{2}$ is contained in a $\left(w_{i}, w_{j}\right)$-segment $P$ of $C$ and subject to this, $P$ is minimal. Since $P$ is a union of a $\left(w_{i}, u_{2}\right)$-segment and a $\left(w_{j}, u_{2}\right)$-segment of $C$, we get that $|P| \geq 8$. There are at least $t-2=\left\lfloor\frac{c}{2}\right\rfloor-3$ segments between two consecutive $w_{\ell}, w_{\ell+1}$ in $C-E(P)$, each of which has length at least 2 . So $c=|C| \geq|P|+2\left(\left\lfloor\frac{c}{2}\right\rfloor-3\right) \geq 8+2\left(\left\lfloor\frac{c}{2}\right\rfloor-3\right) \geq c+1$, a contradiction.

Now suppose that $\left|V\left(B_{1}\right)\right| \geq 3$. So $B_{1}$ is 2-connected. Let $d:=c\left(B_{1}\right)$ and $r:=\left|B_{1}\right|-1$. By the Erdős-Gallai theorem (Theorem 1.1), we have $e\left(B_{1}\right) \leq \frac{d r}{2}$. If $d \geq 2\left\lfloor\frac{c}{2}\right\rfloor-3$, then (c) holds. So we have $d \leq 2\left\lfloor\frac{c}{2}\right\rfloor-4$.

We claim that $e\left(B_{1}-b_{1}, C\right)>\left(\left\lfloor\frac{c}{2}\right\rfloor-1-\frac{d}{2}\right) r$. Suppose for a contradiction that $e\left(B_{1}-b_{1}, C\right) \leq\left(\left\lfloor\frac{c}{2}\right\rfloor-1-\frac{d}{2}\right) r$. Consider $G_{1}:=G-\left(B_{1}-b_{1}\right)$. Since $e\left(B_{1}\right) \leq \frac{d r}{2}$, $e(H)=e\left(B_{1}\right)+e\left(G_{1}-C\right)$ and $e(H, C)=e\left(B_{1}-b_{1}, C\right)+e\left(G_{1}-C, C\right)$, we have

$$
\begin{aligned}
e\left(G_{1}-C\right)+e\left(G_{1}-C, C\right) & =e(H)+e(H, C)-e\left(B_{1}\right)-e\left(B_{1}-b_{1}, C\right) \\
& >\left(\left\lfloor\frac{c}{2}\right\rfloor-1\right) \cdot(n-r-c)=\left(\left\lfloor\frac{c}{2}\right\rfloor-1\right) \cdot\left(\left|V\left(G_{1}\right)\right|-c\right) .
\end{aligned}
$$

As $G_{1}$ is not Hamiltonian (because $\left|G_{1}\right|>|C|$ ), by the choice of $G$, we see that one of $(a),(b)$ and $(c)$ holds in $G_{1}$ and thus in $G$, a contradiction.

Therefore by averaging, there exists a vertex $v_{1} \in V\left(B_{1}-b_{1}\right)$ with $t:=d_{C}\left(v_{1}\right) \geq\left\lfloor\frac{c}{2}\right\rfloor-\frac{d}{2}$. As we have $d \leq 2\left\lfloor\frac{c}{2}\right\rfloor-4$, it follows that $t \geq\left\lfloor\frac{c}{2}\right\rfloor-\frac{d}{2} \geq 2$. Let $w_{1}, w_{2}, \ldots, w_{t}$ be the neighbors of $v_{1}$ on $C$ which appear in a cyclic order. Since $c\left(B_{1}\right)=d$ and $B_{1}$ is 2-connected, $B_{1}$ contains a $\left(v_{1}, b_{1}\right)$-path of length at least $\left\lceil\frac{d}{2}\right\rceil$. So for each $w_{\ell} \in N_{C}\left(v_{1}\right) \backslash\left\{u_{2}\right\}$, there exists a $\left(w_{\ell}, H, u_{2}\right)$-path of length at least $\left\lceil\frac{d}{2}\right\rceil+3$, which in turn implies that any $\left(w_{\ell}, u_{2}\right)$-segment of $C$ has length at least $\left\lceil\frac{d}{2}\right\rceil+3$. This shows that if $t=2$ (and thus $\frac{d}{2}+2 \geq\left\lfloor\frac{c}{2}\right\rfloor$ ), then $c \geq 2\left(\left\lceil\frac{d}{2}\right\rceil+3\right) \geq 2\left(\left\lfloor\frac{c}{2}\right\rfloor+1\right) \geq c+1$, a contradiction. So we have $t \geq 3$. Let $w_{i}, w_{j} \in N_{C}\left(v_{1}\right) \backslash\left\{u_{2}\right\}$ be two vertices such that $u_{2}$ is contained in a $\left(w_{i}, w_{j}\right)$-segment $P$ of $C$ and subject to this, $P$ is minimal. Since $P$ is a union of a $\left(w_{i}, u_{2}\right)$-segment and a $\left(w_{j}, u_{2}\right)$-segment of $C$, we have $|P| \geq 2\left(\left\lceil\frac{d}{2}\right\rceil+3\right)$. Also there are at least $t-2$ segments between two consecutive $w_{\ell}, w_{\ell+1}$ in $C-E(P)$, each of which has length at least 2 . So $c \geq 2(t-2)+2\left(\left\lceil\frac{d}{2}\right\rceil+3\right) \geq 2\left\lfloor\frac{c}{2}\right\rfloor+2 \geq c+1$, a contradiction. This proves Claim 6 .

We now distinguish between the parities of $c$. First assume that $c$ is even. By Claim 2, the average degree of $H$ in $G$ is at least $\frac{c}{2}$. By Lemma 2.3, either $|C| \geq c+1$, or $H$ is a complete graph $K_{\frac{c}{2}-1}$ in which every vertex has the same two neighbors on $C$. Thus, the latter case occurs. Then we have $\left(\begin{array}{c}\frac{c}{2}-1 \\ 2\end{array}\right)+c-2=e(H)+e(H, C)>\left(\frac{c}{2}-1\right)(n-c)=\left(\frac{c}{2}-1\right)^{2}$, 
which implies that $2<c<8$. As $c \geq 5$ is even, we have $c=6$. However, in this case $H$ becomes a $K_{2}$, a contradiction to Claim 5 .

In what follows we consider the case that $c$ is odd. Set $p:=\left|N_{C}(H)\right|$. By Claims 2 and 6, every vertex $v \in V(H)$ has at least $\mu:=\max \left\{\left\lfloor\frac{c}{2}\right\rfloor-p, 2\right\}$ neighbors in $H$. Let $T=\left\{u_{1}, \ldots, u_{t}\right\}$ be a maximum attachment of $H$ to $C$, and $S:=N_{C}(H) \backslash T$, where $t \geq 2$, $s:=|S|$ and $p=s+t$. By Theorem 2.2, there exists a $\left(u_{i}, H, u_{i+1}\right)$-path of length at least $\mu+2$. If $t \geq 3$, then by Lemma 2.4, we have

$$
c \geq t(\mu+2)+2 s=(t-2) \mu+2(\mu+s+t) \geq 2+2\left\lfloor\frac{c}{2}\right\rfloor \geq c+1,
$$

a contradiction. Now we only need to consider the case $t=2$.

Let $T=\left\{u_{1}, u_{2}\right\}$ and $v_{1} u_{1}, v_{2} u_{2}$ be two independent edges for some $v_{1}, v_{2} \in V(H)$. By Lemma 2.4, every vertex in $S$ has a unique neighbor in $H$, which is either $v_{1}$ or $v_{2}$. This shows that for any $u \in V(H) \backslash\left\{v_{1}, v_{2}\right\}, d_{H}(u) \geq \frac{c-1}{2}-2$. By Theorem 2.2, there is a $\left(v_{1}, v_{2}\right)$-path in $H$ of length $\ell \geq \frac{c-1}{2}-2$. So we have a $\left(u_{1}, H, u_{2}\right)$-path of length $\ell+2$. If $|S| \geq 1$ or $\ell \geq \frac{c-1}{2}-1$, then by Lemma 2.4, $c=|C| \geq 2(\ell+2)+2|S| \geq c+1$, a contradiction. So $S=\emptyset$ and $\ell=\frac{c-1}{2}-2$. This implies that every $u \in V(H) \backslash\left\{v_{1}, v_{2}\right\}$ has $d_{H}(u)=\frac{c-1}{2}-2$ and thus is adjacent to both of $u_{1}, u_{2}$.

As $S=\emptyset$, it also holds that $\delta(H) \geq \frac{c-1}{2}-2$. Since $H$ is 2-connected, Dirac's theorem [6] shows that $c(H) \geq \min \{|H|, 2 \delta(H)\} \geq \min \{|H|, c-5\}$. If $c(H) \geq c-4$, then $(c)$ holds. So we have $3 \leq c(H) \leq c-5$ (note that this shows $c \geq 8$ ). This implies that either $c(H)=c-5$, or $c(H)=|H| \leq c-6$. If the latter case holds, then $e(H)+e(H, C) \leq$ $\frac{(n-c)(n-c-1)}{2}+2(n-c) \leq \frac{(n-c)(c-7)}{2}+2(n-c)=\frac{c-3}{2}(n-c)$, a contradiction to (11). So we have $c(H)=|H|=c-5$. Recall that $u_{1}, u_{2}$ are adjacent to all vertices in $H-\left\{v_{1}, v_{2}\right\}$ and $u_{1} v_{1}, u_{2} v_{2} \in E(G)$. There exist two consecutive vertices on the longest cycle in $H$ as the neighbors of $u_{1}, u_{2}$. Using these, we can then find a cycle of length at least $\left\lceil\frac{c}{2}\right\rceil+2+(c-6) \geq c+1$ (as $c \geq 8$ is odd). This final contradiction completes the proof of Theorem 3.2 .

Now we can prove Theorem 3.1 .

Proof of Theorem 3.1. By Theorem 3.2, one of its three cases holds. Since $c \geq 10,(a)$ does not hold. Suppose that $(c)$ holds, i.e., there exists a cycle $C^{\prime}$ with $\left|V\left(C \cap C^{\prime}\right)\right| \leq 1$. Since $G$ is 2-connected, there exist two disjoint paths $P_{1}, P_{2}$ from $x_{1}, x_{2} \in V(C)$ to $y_{1}, y_{2} \in V\left(C^{\prime}\right)$, respectively; moreover, in the case of $\left|V\left(C \cap C^{\prime}\right)\right|=1$, the path $P_{2}$ can be chosen so that $P_{2}$ consists of the single vertex in $V\left(C \cap C^{\prime}\right)$. One can then find a cycle $D$ in the union $C \cup C^{\prime} \cup P_{1} \cup P_{2}$ satisfying that $|D| \geq\left\lceil\frac{c}{2}\right\rceil+\left\lceil\left|C^{\prime}\right| / 2\right\rceil+\left|P_{1}\right|+\left|P_{2}\right|$. If $V\left(C \cap C^{\prime}\right)=\emptyset$, then $|D| \geq\left\lceil\frac{c}{2}\right\rceil+\left(\left\lfloor\frac{c}{2}\right\rfloor-1\right)+2=c+1$, a contradiction; otherwise $\left|V\left(C \cap C^{\prime}\right)\right|=1$, then $|D| \geq\left\lceil\frac{c}{2}\right\rceil+\left\lfloor\frac{c}{2}\right\rfloor+1=c+1$, also a contradiction. This shows that $(c)$ does not hold. Hence, $(b)$ holds, i.e., there exists a vertex $u \in V(G-C)$ with $d_{C}(u)=\left\lfloor\frac{c}{2}\right\rfloor$. It remains to show that $u$ is an isolated vertex in $G-C$. Suppose this is not the case. Then $u$ is contained in a component $R$ of $G-C$ with $|R| \geq 2$. Since $G$ is 2-connected, there exists a vertex $v \in V(R-u)$ with a neighbor in $V(C)$. Using this, one can easily find a cycle of length at least $c+1$. This finishes the proof of Theorem 3.1.

\subsection{Proof of Theorem 1.12}

To prove Theorem 1.12, in view of Theorem 3.1, it suffices to show the following lemma. 
Lemma 3.3. Let $G$ be a 2-connected non-Hamiltonian graph on $n$ vertices and $C$ be a longest cycle in $G$ of length c. Suppose that there exists an isolated vertex $u$ in $G-C$ with $d_{C}(u)=\left\lfloor\frac{c}{2}\right\rfloor$.

- If $c$ is even, then $G \subseteq W_{n,\left\lfloor\frac{c}{2}\right\rfloor, c}$.

- If $c \geq 9$ is odd, then $G \subseteq W_{n,\left\lfloor\frac{c}{2}\right\rfloor, c}$ or $G$ is a subgraph of a member of $\mathcal{X}_{n, c} \cup \mathcal{Y}_{n, c}$.

Proof. Throughout this proof, let $N:=N_{C}(u)$.

We first consider the case that $c$ is even. Let $C=x_{1} x_{2} \ldots x_{c} x_{1}$. We may assume that $N=\left\{x_{1}, x_{3}, \ldots, x_{c-1}\right\}$. Consider any component $R$ in $G-C$ with $u \notin V(R)$. As $G$ is 2-connected, $\left|N_{C}(R)\right| \geq 2$. We also have that $C-N$ consists of isolated vertices and $\left|N_{C}(R) \backslash N\right| \leq 1$ (otherwise one can easily find a cycle longer than $C$ using Posa's rotation technique). Suppose that there exist some vertices say $x_{2} \in N_{C}(R) \backslash N$ and $x_{i} \in$ $N_{C}(R) \cap N$. We assume that $i \notin\{1,3\}$ (as otherwise there is a cycle longer than $C$ ). There exists an $\left(x_{2}, R, x_{i}\right)$-path $P$ of length at least 2, then $P \cup\left(C-x_{i+1}-x_{2} x_{3}\right) \cup\left\{u x_{3}, u x_{i+2}\right\}$ forms a cycle of length at least $c+1$, a contradiction. Thus $N_{C}(R) \subseteq N$. If $|R| \geq 2$, then there exist distinct $x_{i}, x_{j} \in N_{C}(R) \cap N$ and an $\left(x_{i}, R, x_{j}\right)$-path $Q$ of length at least 3 . One can find a longer cycle easily if the distance between $x_{i}$ and $x_{j}$ on $C$ is two; otherwise, $Q \cup\left(C-\left\{x_{i+1}, x_{j+1}\right\}\right) \cup\left\{u x_{i+2}, u x_{j+2}\right\}$ forms a cycle longer than $C$. This shows that $|R|=1$ and $N_{C}(R) \subseteq N$ for any component $R$ in $G-C$. Therefore indeed $G$ is a subgraph of $W_{n, \frac{c}{2}, c}$ when $c$ is even.

From now on we assume that $c \geq 9$ is odd. Let $c:=2 \alpha+1$ and $C=x_{1} x_{2} \ldots x_{2 \alpha+1} x_{1}$, where $\alpha \geq 4$. We may assume that $N=N_{C}(u)=\left\{x_{1}, x_{3}, \ldots, x_{2 \alpha-1}\right\}$. First we observe an easy fact that $C-N$ consists of a unique edge $x_{2 \alpha} x_{2 \alpha+1}$ and isolated vertices. Next we determine the structures of all components $R$ in $G-C$.

Claim. Any component $R$ in $G-C$ is of one of the following three types:

(i) $|R|=1$ and $N_{C}(R) \subseteq N_{C}(u)$;

(ii) $|R|=1$ and $N_{C}(R)=\left\{x_{2 \alpha-1}, x_{2 \alpha+1}\right\}$ or $N_{C}(R)=\left\{x_{1}, x_{2 \alpha}\right\}$;

(iii) $R$ is an induced star, which is $\left\{x_{1}, x_{2 \alpha-1}\right\}$-feasible 8

Proof of Claim. First assume that there are two vertices $a, b$ in $N_{C}(R) \backslash N$. Then there exists an $(a, R, b)$-path $P$ of length at least 2 . If $\{a, b\}=\left\{x_{2 \alpha}, x_{2 \alpha+1}\right\}$, then $\left(C-x_{2 \alpha} x_{2 \alpha+1}\right) \cup P$ forms a cycle of length at least $c+1$. Otherwise, we have either $a^{+}, b^{+} \in N$ or $a^{-}, b^{-} \in N$. We may assume the former case occurs. Then $P \cup\left(C-\left\{a a^{+}, b b^{+}\right\}\right) \cup\left\{u a^{+}, u b^{+}\right\}$forms a cycle of length at least $c+1$, a contradiction.

Now assume that $N_{C}(R) \subseteq N$. If $|R|=1$, then $R$ is of type (i). So $|R| \geq 2$. As $G$ is 2-connected, there exist $x_{2 i-1}, x_{2 j-1} \in N_{C}(R)$ and an $\left(x_{2 i-1}, R, x_{2 j-1}\right)$-path $P$ of length at least 3. Suppose that $\left\{x_{2 i-1}, x_{2 j-1}\right\} \neq\left\{x_{1}, x_{2 \alpha-1}\right\}$. If the distance between $x_{2 i-1}$ and $x_{2 j-1}$ on $C$ is two, then it is easy to find a cycle of length at least $c+1$; otherwise, since $\alpha \geq 4$, without loss of generality we may assume that $1 \leq 2 j-1<2 j+1<2 i-1<$ $2 i+1 \leq 2 \alpha-1$, then $P \cup\left(C-\left\{x_{2 j}, x_{2 i}\right\}\right) \cup\left\{u x_{2 j+1}, u x_{2 i+1}\right\}$ forms a cycle of length at least $c+1$, a contradiction. This shows that $N_{C}(R)=\left\{x_{1}, x_{2 \alpha-1}\right\}$. If there exists an $\left(x_{1}, R, x_{2 \alpha-1}\right)$-path $P$ of length at least 4 , then $\left(C-\left\{x_{2 \alpha}, x_{2 \alpha+1}\right\}\right) \cup P$ is a cycle of length at least $c+1$. Hence, all $\left(x_{1}, R, x_{2 \alpha-1}\right)$-paths in $G\left[R \cup N_{C}(R)\right]$ are of length 3. This forces $R$ to be an induced star, and moreover, if $|R| \geq 3$, then all leaves of $R$ are only adjacent to the same vertex in $\left\{x_{1}, x_{2 \alpha-1}\right\}$. So $R$ is of type (iii).

\footnotetext{
${ }^{8}$ Recall the definition of $\{a, b\}$-feasible from Subsection 1.2.
} 
It remains to consider that $\left|N_{C}(R) \backslash N\right|=1$. As $G$ is 2-connected, there exists some $x_{2 j-1} \in N_{C}(R) \cap N$. Let $P$ be an $\left(x_{2 j-1}, R, N_{C}(R) \backslash N\right)$-path of length at least 2 . Let us first consider that $2 \leq j \leq \alpha-1$. If $N_{C}(R) \backslash N=\left\{x_{2 i}\right\}$, where $1 \leq i \leq \alpha$, then we may assume that $x_{2 j-1}$ and $x_{2 i}$ are not adjacent (as otherwise there is a longer cycle). By symmetry, we may also assume $2 j-1<2 i-1<2 i$. Thus we have $1 \leq 2 j-3<2 j-1<2 i-1<2 i \leq 2 \alpha$. Then $P \cup\left(C-x_{2 j-2}-x_{2 i-1} x_{2 i}\right) \cup\left\{x_{2 j-3} u, u x_{2 i-1}\right\}$ forms a cycle of length at least $c+1$, a contradiction. So, $N_{C}(R) \backslash N=\left\{x_{2 \alpha+1}\right\}$. Then, $\left(C-x_{2 j}-x_{2 \alpha+1} x_{1}\right) \cup P \cup\left\{x_{1} u, u x_{2 j+1}\right\}$ is a cycle of length at least $c+1$, again a contradiction. Hence, we have that $j \in\{1, \alpha\}$. By symmetry, we may just consider $j=1$. In this case, $x_{1} \in N_{C}(R) \cap N$ (so clearly $\left.x_{2}, x_{2 \alpha+1} \notin N_{C}(R)\right)$ and we claim that $N_{C}(R) \backslash N=\left\{x_{2 \alpha}\right\}$. Suppose for a contradiction that $N_{C}(R) \backslash N=\left\{x_{2 i}\right\}$ for $2 \leq i \leq \alpha-1$. Then $\left(C-x_{2}-x_{2 i} x_{2 i+1}\right) \cup P \cup\left\{x_{3} u, u x_{2 i+1}\right\}$ forms a cycle of length at least $c+1$, a contradiction. This shows that $N_{C}(R)=\left\{x_{1}, x_{2 \alpha}\right\}$. If $|R| \geq 2$, then $P$ can be chosen to be a path of length at least 3 and the cycle $P \cup\left(C-x_{2 \alpha+1}\right)$ contradicts the maximality of $C$. Therefore, $|R|=1$ and $N_{C}(R)=\left\{x_{1}, x_{2 \alpha}\right\}$. So $R$ is of type (ii). This proves the claim.

We show that all components $R$ in $G-C$ of type (ii) have the same two neighbors in $C$ (say $N_{C}(R)=\left\{x_{1}, x_{2 \alpha}\right\}$ ). Otherwise there are two components in $G-C$ of type (ii), say $R_{1}=\left\{v_{1}\right\}$ and $R_{2}=\left\{v_{2}\right\}$, such that $N_{C}\left(v_{1}\right)=\left\{x_{2 \alpha-1}, x_{2 \alpha+1}\right\}$ and $N_{C}\left(v_{2}\right)=\left\{x_{1}, x_{2 \alpha}\right\}$, then $G\left[V(C) \cup\left\{v_{1}, v_{2}\right\}\right]$ contains a cycle of length $c+2$, a contradiction.

If all components in $G-C$ are of type (i), then as $N^{+}$is independent, we have $G \subseteq W_{n,\left\lfloor\frac{c}{2}\right\rfloor, c}$. So there exists at least one component in $G-C$ of type (ii) or (iii).

Suppose that there is no component in $G-C$ of type (iii). Then there exists some component in $G-C$, say $\{v\}$, of type (ii). So we can assume $N_{C}(v)=\left\{x_{1}, x_{2 \alpha}\right\}$. We show that $N_{G}\left(x_{2 \alpha+1}\right)=\left\{x_{1}, x_{2 \alpha}\right\}$. To see this, consider $C^{\prime}:=\left(C-\left\{x_{1} x_{2 \alpha+1}, x_{2 \alpha+1} x_{2 \alpha}\right\}\right) \cup$ $\left\{x_{1} v, v x_{2 \alpha}\right\}$, which also is a longest cycle in $G$. Then $x_{2 \alpha+1}$ is contained in a component $R^{\prime}$ in $G-C^{\prime}$. As $N_{C^{\prime}}\left(R^{\prime}\right) \supseteq\left\{x_{1}, x_{2 \alpha}\right\}$, by the Claim, $R^{\prime}$ must be of type (ii) and thus we have $N_{G}\left(x_{2 \alpha+1}\right)=\left\{x_{1}, x_{2 \alpha}\right\}$. Let $J_{1}$ (resp. $J_{2}$ ) be the set of all vertices in components in $G-C$ of type (i) (resp. type (ii)). Now set $A:=N, B:=N^{+} \cup J_{1}$ and $X:=\left\{x_{2 \alpha+1}\right\} \cup J_{2}$. Then both $B$ and $X$ are stable and for any $w \in X, N_{G}(w)=\left\{x_{1}, x_{2 \alpha}\right\}$. This shows that $G$ is a subgraph of some graph from $\mathcal{X}_{n, c}$.

Now we assume that there exists some component $R$ in $G-C$ of type (iii). Let $J_{1}, J_{2}, J_{3}$ be the sets of all vertices in components in $G-C$ of type (i), (ii), (iii), respectively. Set $A:=$ $N, B:=\left\{x_{2}, x_{4}, \ldots, x_{2 \alpha-2}\right\} \cup J_{1}$, and $Y:=\left\{x_{2 \alpha}, x_{2 \alpha+1}\right\} \cup J_{2} \cup J_{3}$. Clearly $B$ is stable. Since every vertex $v \in J_{2}$ satisfies $N_{C}(v)=\left\{x_{1}, x_{2 \alpha}\right\}$, we see that $G\left[\left\{x_{2 \alpha}, x_{2 \alpha+1}\right\} \cup J_{2}\right]$ induces a star, say $S$, with the center $x_{2 \alpha}$. If we can show that $S$ is $\left\{x_{1}, x_{2 \alpha-1}\right\}$-feasible, then $G$ is a subgraph of some graph from $\mathcal{Y}_{n, c}$ (note that $G[Y]$ has at least two stars). To show this, we note that there exists an edge $x y$ in $R$ such that $C^{\prime}:=\left(C-\left\{x_{2 \alpha}, x_{2 \alpha+1}\right\}\right) \cup\left\{x_{1} x, x y, y x_{2 \alpha-1}\right\}$ is a longest cycle in $G$. Then $S=G\left[\left\{x_{2 \alpha}, x_{2 \alpha+1}\right\} \cup J_{2}\right]$ is contained in a component $R^{\prime}$ in $G-C^{\prime}$. By the Claim, $R^{\prime}$ must be of type (iii), i.e., $R^{\prime}$ (and thus $S$ ) is $\left\{x_{1}, x_{2 \alpha-1}\right\}$-feasible. This proves Lemma 3.3 .

We have completed the proof of Theorem 1.12. 


\section{Stability from many edges spanned in a long cycle}

In this section, we prove the following strengthened version of Theorem 1.13, where the longest cycle in Theorem 1.13 is generalized to a locally maximal cycle.

Recall that $h(n, k)=\left(\begin{array}{c}n-k \\ 2\end{array}\right)+k(k-1)$.

Theorem 4.1. Let $G$ be a 2-connected graph on $n$ vertices with $\delta(G) \geq k$ and $C$ be a locally maximal cycle in $G$ of length $c \in[6, n-1]$. If $e(G)>\max \left\{f(n, k+1, c), f\left(n,\left\lfloor\frac{c}{2}\right\rfloor-1, c\right)\right\}$ and $e(G[C])>h\left(c+1,\left\lfloor\frac{c}{2}\right\rfloor-1\right)$, then either $G \subseteq W_{n,\left\lfloor\frac{c}{2}\right\rfloor, c}$, or $\bar{G} \in\left\{W_{n, k, c}, Z_{n, k, c}\right\}$, where $\bar{G}$ is the $C$-closure of $G$.

We will reduce Theorem 4.1 to the following three lemmas, which are needed when dealing with the two situations arising from Lemma 2.11.

Lemma 4.2. Let $G_{c}$ be a Hamiltonian graph on $c \geq 6$ vertices. Further suppose that $G_{c}$ is $(c+1)$-closed and non-Hamiltonian-connected with $e\left(G_{c}\right)>h\left(c+1,\left\lfloor\frac{c}{2}\right\rfloor-1\right)$. If there exist $\left\lfloor\frac{c}{2}\right\rfloor-1$ vertices of degree at most $\left\lfloor\frac{c}{2}\right\rfloor$ in $G_{c}$, then $G_{c}=W_{c,\left\lfloor\frac{c}{2}\right\rfloor, c}$.

Lemma 4.3. Let $G$ be a 2-connected graph on $n$ vertices and $C$ be a locally maximal cycle in $G$ of length $c \leq n-1$. Suppose that $e(G)>\max \left\{f(n, k+1, c), f\left(n,\left\lfloor\frac{c}{2}\right\rfloor-1, c\right)\right\}$. If $G[C]$ contains a subset $S$ of $s-1$ vertices of degree at most $s$ in $G[C]$ for some integer $2 \leq s \leq\left\lfloor\frac{c}{2}\right\rfloor-1$ such that $G[C]-S$ is a clique, then $2 \leq s \leq k$ and the clique number of $G[C]$ is at least $c-k+1$.

Lemma 4.4. Let $G$ be a 2-connected graph on $n$ vertices with $\delta(G) \geq k$ and $C$ be a locally maximal cycle in $G$ of length $c \leq n-1$. If the clique number of $G[C]$ is at least $c-k+1$, then $G \in\left\{W_{n, k, c}, Z_{n, k, c}\right\}$.

This reduction will be done in Subsection 4.1. We then prove these lemmas in Subsections 4.2, 4.3 and 4.4, respectively.

\subsection{Reducing Theorem 4.1 to the lemmas}

Proof of Theorem 4.1. (Assuming Lemmas 4.2, 4.3 and 4.4.) Let $G, C$ be as in Theorem 4.1. Let $\bar{G}$ be the $C$-closure of $G$. Since $G \subseteq \bar{G}$, we see that $\bar{G}$ is 2-connected with $\delta(\bar{G}) \geq k$ and $e(\bar{G}) \geq e(G)$. By Lemma 2.7, we see that the cycle $C$ remains a locally maximal cycle of length $c$ in $\bar{G}$. By Lemma $2.8, \bar{G}[C]$ is non-Hamiltonian-connected. It is also clear that $\bar{G}[C]$ is $(c+1)$-closed and $e(\bar{G}[C]) \geq e(G[C])>h\left(c+1,\left\lfloor\frac{c}{2}\right\rfloor-1\right)$.

Applying Lemma 2.11 (with $\delta=1$ ) to $\bar{G}[C]$, we see that one of the following holds:

(i) $\bar{G}[C]$ contains a subset of $\left\lfloor\frac{c}{2}\right\rfloor-1$ vertices of degree at most $\left\lfloor\frac{c}{2}\right\rfloor$ in $\bar{G}[C]$, or

(ii) $\bar{G}[C]$ contains a subset $S$ of $s-1$ vertices of degree at most $s$ in $\bar{G}[C]$ for some $2 \leq s \leq\left\lfloor\frac{c}{2}\right\rfloor-2$ such that $\bar{G}[C]-S$ is a clique.

Suppose that (i) holds. Then by Lemma 4.2 (applied to $\bar{G}[C]$ ), we have $\bar{G}[C]=W_{c,\left\lfloor\frac{c}{2}\right\rfloor, c}$. Let $B \subseteq V(C)$ consist of all vertices of degree $c-1$ in $\bar{G}[C]$. We observe that for any two vertices $x, y \in V(C)$, if $\{x, y\} \nsubseteq B$, then there is a Hamiltonian path from $x$ to $y$ in $\bar{G}[C]$. Thus, for any component $R$ in $\bar{G}-C$, if $N_{C}(R) \nsubseteq B$, then there is a cycle $C^{\prime}$ longer than $C$ with $\left|E\left(C^{\prime}\right) \cap E(C, G-C)\right| \leq 2$, a contradiction. So, we have $N_{C}(R) \subseteq B$. Furthermore, for any $\{x, y\} \subseteq B$, there is an $(x, y)$-path of length at least $c-2$ in $\bar{G}[C]$. If $|V(R)| \geq 2$, as 
$\bar{G}$ is 2-connected, we can find a cycle $C^{\prime}$ longer than $C$ with $\left|E\left(C^{\prime}\right) \cap E(C, G-C)\right| \leq 2$, a contradiction. Hence, for any component $R$ in $\bar{G}-C$, we have $|V(R)|=1$ and $N_{C}(R) \subseteq B$. This implies that $G \subseteq \bar{G} \subseteq W_{n,\left\lfloor\frac{c}{2}\right\rfloor, c}$.

So we may assume that (ii) holds. By Lemma 4.3 (applied to $\bar{G}$ and $C$ ), we get that the clique number of $\bar{G}[C]$ is at least $c-k+1$. By Lemma 4.4, this shows that $\bar{G} \in\left\{W_{n, k, c}, Z_{n, k, c}\right\}$, completing the proof of Theorem 4.1.

\subsection{Proof of Lemma 4.2}

Proof. Throughout this proof, define $\alpha:=\left\lfloor\frac{c}{2}\right\rfloor, e:=e\left(G_{c}\right), A:=\left\{u \in V\left(G_{c}\right): d(u) \leq\right.$ $\alpha$, and $B:=V\left(G_{c}\right) \backslash A$. Since $G_{c}$ is $(c+1)$-closed, $B$ induces a clique. Let $V\left(G_{c}\right)=$ $\left\{u_{1}, u_{2}, \ldots, u_{c}\right\}$ and $f_{1} \leq f_{2} \leq \ldots \leq f_{c}$ be the degree sequence of $G_{c}$ such that $d\left(u_{i}\right)=f_{i}$ for every $1 \leq i \leq c$. There are $\alpha-1$ vertices of degree at most $\alpha$ in $G_{c}$, in other words, we have $f_{\alpha-1} \leq \alpha$.

We establish some facts to be used later. The first two facts are straightforward.

Fact 1. If $G_{c}$ has $t$ vertices of degree at most $r$, then $e\left(G_{c}\right) \leq t r+\left(\begin{array}{c}c-t \\ 2\end{array}\right)$.

Fact 2. We have

$h\left(1+c,\left\lfloor\frac{c}{2}\right\rfloor-1\right)=\frac{1}{2}\left\lceil\frac{c}{2}\right\rceil^{2}+\frac{3}{2}\left\lceil\frac{c}{2}\right\rceil+\left\lfloor\frac{c}{2}\right\rfloor^{2}-3\left\lfloor\frac{c}{2}\right\rfloor+3= \begin{cases}\frac{3 c^{2}}{8}-\frac{3 c}{4}+3 & \text { if } c \text { is even } \\ \frac{3 \alpha^{2}}{2}-\frac{\alpha}{2}+5 & \text { if } c \text { is odd. }\end{cases}$

Fact 3. $f_{\alpha-1}=\alpha$ and $f_{c-\alpha} \leq c-\alpha$. Thus, when $c$ is even, we have $f_{\frac{c}{2}}=\frac{c}{2}$.

Proof. Suppose for a contradiction that $f_{\alpha-1} \leq \alpha-1$. By Facts 1 and 2 , we have the following: if $c$ is even, then $e \leq\left(\frac{c}{2}-1\right)^{2}+\left(\begin{array}{c}\frac{c}{2}+1 \\ 2\end{array}\right)=\frac{3 c^{2}}{8}-\frac{3 c}{4}+1<h\left(1+c,\left\lfloor\frac{c}{2}\right\rfloor-1\right)<e$, a contradiction; if $c$ is odd, then $e \leq\left(\left\lfloor\frac{c}{2}\right\rfloor-1\right)^{2}+\left(\begin{array}{c}\left\lceil\frac{c}{2}\right\rceil+1 \\ 2\end{array}\right)=\frac{3 \alpha^{2}}{2}-\frac{\alpha}{2}+2<h\left(1+c,\left\lfloor\frac{c}{2}\right\rfloor-1\right)<e$, also a contradiction. Thus, $f_{\alpha-1}=\alpha$.

Suppose that $f_{c-\alpha} \geq c-\alpha+1$. First assume that $c$ is even. As $f_{\alpha}=f_{c-\alpha} \geq \alpha+1$, we have $d\left(u_{\alpha}\right)+d\left(u_{\alpha-1}\right) \geq c+1$, so $u_{\alpha-1}$ is adjacent to all vertices in $\left\{u_{\alpha}, u_{\alpha+1}, \ldots, u_{c}\right\}$. This implies that $d\left(u_{\alpha-1}\right)=f_{\alpha-1} \geq \alpha+1$, a contradiction. Now consider that $c$ is odd. As $f_{\alpha+1} \geq \alpha+2, u_{\alpha-1}$ is adjacent to all vertices in $\left\{u_{\alpha+1}, u_{\alpha+2}, \ldots, u_{2 \alpha+1}\right\}$, thus $f_{\alpha-1}=d\left(u_{\alpha-1}\right) \geq \alpha+1$, again a contradiction. This finishes the proof.

Fact 4. For every vertex $u \in V\left(G_{c}\right)$, either $d(u)=c-1$ or $2 \leq d(u) \leq c-3$.

Proof. Suppose for a contradiction that there exists a vertex $u$ with $d(u)=c-2$. Then there exists a vertex $v$ not adjacent to $u$. As $G_{c}$ is $(c+1)$-closed, we have $d(u)+d(v) \leq c$, implying that $d(v) \leq 2$. Since $G_{c}$ is Hamiltonian, $d(v)=2$. When $c$ is even, we have $e \leq 2+\sum_{j=2}^{\frac{c}{2}} f_{j}+e\left(G\left[\left\{u_{\frac{c}{2}+1}, \ldots, u_{c}\right\}\right]\right) \leq 2+\left(\frac{c}{2}-1\right) \frac{c}{2}+\left(\frac{c}{2}\right)=\frac{3 c^{2}}{8}-\frac{3 c}{4}+2<h(1+$ $\left.c,\left\lfloor\frac{c}{2}\right\rfloor-1\right)<e$, a contradiction. If $c$ is odd, then $e \leq 2+\sum_{j=2}^{\alpha+1} f_{j}+e\left(G\left[\left\{u_{\alpha+2}, \ldots, u_{c}\right\}\right]\right) \leq$ $2+(\alpha-2) \alpha+2(\alpha+1)+\left(\begin{array}{l}\alpha \\ 2\end{array}\right)=\frac{3 \alpha^{2}}{2}-\frac{\alpha}{2}+4<e$, a contradiction. This proves Fact 4.

Fact 5. If it exists, let $u \in B$ be the vertex such that $d(u):=c-i$ is maximum over all vertices in $B$ with degree at most $c-2$, where $3 \leq i \leq c-\alpha-1$. If $|B| \geq i$, then $e\left(G_{c}\right) \leq i^{2}-\frac{i}{2}(|B|+\alpha+2)+\frac{\alpha}{2}(c+1)+\frac{|B|}{2}(c-\alpha)$. 
Proof. Since $B$ induces a clique, all $i-1$ non-neighbors of $u$ are in $A$. Let $A^{\prime}$ be the subset of $A$ consisting of such $i-1$ vertices. Since $G_{c}$ is $(c+1)$-closed, every vertex $x \in A^{\prime}$ has $2 \leq d(x) \leq i$. Choose a fixed vertex $x \in A^{\prime}$ and let $B^{\prime} \subseteq B$ be the set of all non-neighbors of $x$ in $B$. Then $\left|B^{\prime}\right| \geq|B|-i$, and for any $y \in B^{\prime}$, we have $d(y) \leq c-d(x) \leq c-2$. By Fact 4 , we see that any $y \in B^{\prime}$ has degree at most $c-3$, therefore, by the choice of $u$, $d(y) \leq d(u)=c-i$. Now we get that

$$
\begin{aligned}
e\left(G_{c}\right) & =\frac{1}{2} \sum_{i=1}^{c} f_{i}=\frac{1}{2}\left(\sum_{v \in A^{\prime}} d(v)+\sum_{v \in A \backslash A^{\prime}} d(v)+\sum_{v \in B^{\prime}} d(v)+\sum_{v \in B \backslash B^{\prime}} d(v)\right) \\
& \leq \frac{1}{2}\left((i-1) i+(|A|-i+1) \alpha+\left|B^{\prime}\right|(c-i)+\left(|B|-\left|B^{\prime}\right|\right)(c-1)\right) \\
& \leq i^{2}-i(|B|+\alpha+2) / 2+\alpha(c+1) / 2+|B|(c-\alpha) / 2,
\end{aligned}
$$

where the last inequality holds because $|A|=c-|B|$ and $\left|B^{\prime}\right| \geq|B|-i$.

We divide the rest of the proof into two cases depending on the parity of $c$.

Case 1. $c$ is even.

In this case, we have $f_{\frac{c}{2}-1}=f_{\frac{c}{2}}=\frac{c}{2}$. First we claim that $f_{\frac{c}{2}+2} \geq \frac{c}{2}+1$. Otherwise, $f_{\frac{c}{2}+2}=\frac{c}{2}$, then $|A| \geq \frac{c}{2}+2$ and $|B| \leq \frac{c}{2}-2$. This implies that $e=\frac{1}{2} \sum_{j=1}^{c} f_{j} \leq$ $\frac{1}{2}\left(\left(\frac{c}{2}+2\right) \frac{c}{2}+\left(\frac{c}{2}-2\right)(c-1)\right)=\frac{3 c^{2}}{8}-\frac{3 c}{4}+1<h\left(1+c,\left\lfloor\frac{c}{2}\right\rfloor-1\right)<e$, a contradiction.

Next we show that $f_{\frac{c}{2}+1} \geq \frac{c}{2}+1$. Suppose not. Then we have $f_{\frac{c}{2}+1}=\frac{c}{2}$ and $|B|=\frac{c}{2}-1$. Suppose there exists some vertex in $B$ with degree at most $c-2$. By Fact 5 , there exists some $3 \leq i \leq \frac{c}{2}-1$ such that $e \leq i^{2}-\frac{i}{2}(c+1)+\frac{3 c^{2}}{8} \leq \frac{3 c^{2}}{8}-\frac{3 c}{4}+3<e$, a contradiction. To see why the second inequality holds, let $f(i):=i^{2}-\frac{i}{2}(c+1)$; then we have $f(i) \leq$ $\max \left\{f(3), f\left(\frac{c}{2}-1\right)\right\}$ and it is routine to check that as $c \geq 6$, this is at most $-\frac{3 c}{4}+\frac{3}{2}$. Hence we may assume that every vertex in $B$ has degree $c-1$. Let $H$ be the spanning subgraph of $G_{c}$ consisting of all edges in $E(B) \cup(A, B)$. As $e(H)=\left(\frac{c}{2}+1\right)\left(\frac{c}{2}-1\right)+\left(\begin{array}{c}\frac{c}{2}-1 \\ 2\end{array}\right)=\frac{3 c^{2}}{8}-\frac{3 c}{4}$ and $e>\frac{3 c^{2}}{8}-\frac{3 c}{4}+3$, we see that $E(A)$ has at least 4 edges. Observe that every vertex in $A$ has degree at most $c / 2$ in $G_{c}$ and is already adjacent to the $c / 2-1$ vertices in $B$. This shows that there exists a matching of size at least 4 in $A$. One can check that the subgraph obtained from $H$ by adding a matching of size 3 in $A$ is already Hamiltonian-connected. So is the host graph $G_{c}$. But this is a contradiction. This proves that $f_{\frac{c}{2}+1} \geq \frac{c}{2}+1$ and thus $|A|=|B|=\frac{c}{2}$.

Lastly, we show that any vertex $u \in B$ has degree $c-1$. Suppose for a contradiction that there exists a vertex $u \in V(B)$ with $d(u) \leq c-2$. By Fact 5 , there exists some $3 \leq i \leq \frac{c}{2}-1$ such that $e \leq i^{2}-\frac{i}{2}(c+2)+\frac{3 c^{2}}{8}+\frac{c}{4} \leq \frac{3 c^{2}}{8}-\frac{3 c}{4}+3<e$, where the second inequality can be verified similarly as above for $c \geq 6$, a contradiction.

Now, we see that $B$ induces a clique $K_{\frac{c}{2}}$ and $(A, B)$ is complete bipartite. As every vertex in $A$ has degree at most $\frac{c}{2}$, we see that $E(A)$ contains no edge. This shows $G_{c}=$ $W_{c, \frac{c}{2}, c}$, completing the proof of Case 1 .

Case 2. $c$ is odd.

Let $H$ be the spanning subgraph of $G_{c}$ consisting of all edges in $E(B) \cup(A, B)$. In this case, $c=2 \alpha+1$, where $\alpha \geq 3$. By Facts 1 and 2 , we have $\frac{3 \alpha^{2}}{2}-\frac{\alpha}{2}+5<e \leq$ $(\alpha-1) \alpha+\left(\begin{array}{c}\alpha+2 \\ 2\end{array}\right)=\frac{3 \alpha^{2}}{2}+\frac{\alpha}{2}+1$; by Fact $3, f_{\alpha-1}=\alpha$ and $f_{\alpha+1} \leq \alpha+1$. 
We show in a sequence of claims that $f_{\alpha}=f_{\alpha+1}=\alpha+1$. First we show $f_{\alpha+3} \geq \alpha+1$. Otherwise, $f_{\alpha+3} \leq \alpha$. Then $|A| \geq \alpha+3$ and $|B| \leq \alpha-2$, from which we derive a contradiction that $\left.e \leq \frac{1}{2}((\alpha+3) \alpha)+(\alpha-2)(2 \alpha)\right)=\frac{3 \alpha^{2}-\alpha}{2}<e$.

Next we show that $f_{\alpha+2} \geq \alpha+1$. Suppose not. Then $f_{\alpha-1}=f_{\alpha}=f_{\alpha+1}=f_{\alpha+2}=\alpha$. So $B=\left\{u_{\alpha+3}, \ldots, u_{2 \alpha+1}\right\}$ and $|B|=\alpha-1$. Suppose that there are vertices in $B$ with degree at most $c-2$. Let $u \in B$ be such a vertex with maximum degree $d(u)=c-i$, where $3 \leq i \leq \alpha$. If $3 \leq i \leq \alpha-1$, then by Fact 5 , we have $e \leq i^{2}-\frac{i}{2}(2 \alpha+1)+\left(\alpha^{2}+\alpha\right)+\frac{\alpha^{2}-1}{2} \leq$ $\frac{3 \alpha^{2}}{2}-\frac{\alpha}{2}+5<e$, where the second inequality holds since $i^{2}-\frac{i}{2}(2 \alpha+1)$ takes the maximum at $i=3$ or $\alpha-1$. This is a contradiction. So $i=\alpha$, that is, $d(u)=\alpha+1$. Then $e \leq \frac{1}{2}((\alpha+2) \alpha+(\alpha+1)+(\alpha-2)(2 \alpha))=\frac{1}{2}\left(3 \alpha^{2}-\alpha+1\right)<\frac{3 \alpha^{2}}{2}-\frac{\alpha}{2}+5<e$, again a contradiction. Now we may assume that every vertex in $B$ has degree $2 \alpha$. So $(A, B)$ is complete bipartite and thus every vertex in $A$ has degree $\alpha-1$ in the subgraph $H$ defined above. By the definition of $A$, every vertex in $A$ has degree at most $\alpha$ in $G_{c}$. This shows that $E(A)$ must be a matching (if not empty). Since $e(H)=\left(\begin{array}{c}\alpha-1 \\ 2\end{array}\right)+(\alpha-1)(\alpha+2)=$ $\frac{3 \alpha^{2}}{2}-\frac{\alpha}{2}-1$ and $e>\frac{3 \alpha^{2}}{2}-\frac{\alpha}{2}+5$, we see that $E(A)$ forms a matching of size at least 7. One can check that the subgraph obtained from $H$ by adding a matching of size 4 in $A$ is Hamiltonian-connected, so the host graph $G_{c}$ is also Hamiltonian-connected, a contradiction. This proves $f_{\alpha+2} \geq \alpha+1$.

We also claim that $f_{\alpha+1}=\alpha+1$. Suppose not. Then we have $f_{\alpha-1}=f_{\alpha}=f_{\alpha+1}=\alpha$ and $B=\left\{u_{\alpha+2}, \ldots, u_{2 \alpha+1}\right\}$. So $|B|=\alpha$. First suppose that every vertex $b \in B$ has degree $c-1$. Then the subgraph $H$ is just a vertex-disjoint union of a clique $K_{\alpha}$ and an independent set of size $\alpha+1$, with a complete bipartite subgraph between the two parts. So $\frac{3 \alpha^{2}}{2}+\frac{\alpha}{2}+1 \geq e \geq e(H)=\alpha(\alpha+1)+\left(\begin{array}{l}\alpha \\ 2\end{array}\right)=\frac{3 \alpha^{2}}{2}+\frac{\alpha}{2}$, which implies that $E(A)$ has at most one edge. Thus, $G_{c}=H$ or $W_{c,\left\lfloor\frac{c}{2}\right\rfloor, c}$. But for the former case, $G_{c}=H$ is not Hamiltonian. Hence in this case, we prove $G_{c}=W_{c,\left\lfloor\frac{c}{2}\right\rfloor, c}$. Now we may assume that there are vertices in $B$ of degree at most $c-2$. Let $u \in B$ be such a vertex with maximum degree $d(u)=c-i$, where $3 \leq i \leq \alpha$. If $3 \leq i \leq \alpha-1$, by Fact 5 we have $e \leq i^{2}-i(\alpha+1)+\frac{3}{2}\left(\alpha^{2}+\alpha\right) \leq \frac{3 \alpha^{2}}{2}-\frac{\alpha}{2}+5<e$, where the second inequality holds since $i^{2}-i(\alpha+1)$ takes the maximum at $i=3$ or $\alpha-1$. So we must have $i=\alpha$. This shows that for any $b \in B$, either $d(b)=2 \alpha$ or $d(b)=\alpha+1$. If there exist at least two vertices in $B$ of degree $\alpha+1$, then $e \leq \frac{1}{2}((\alpha+1) \alpha+2(\alpha+1)+(\alpha-2)(2 \alpha))=\frac{3 \alpha^{2}}{2}-\frac{\alpha}{2}+1<e$, a contradiction. So $B$ contains $\alpha-1$ vertices of degree $2 \alpha$ and a vertex say $u$ of degree $\alpha+1$. Every $x \in A$ has at least $\alpha-1$ neighbors in $B$; this shows that $E(A)$ is a matching. Note that the vertex $u$ has two neighbors in $A$. So $e(H)=\left(\begin{array}{l}\alpha \\ 2\end{array}\right)+2+(\alpha-1)(\alpha+1)=\frac{3 \alpha^{2}}{2}-\frac{\alpha}{2}+1$. This, together with $e>\frac{3 \alpha^{2}}{2}-\frac{\alpha}{2}+5$, shows that $E(A)$ is a matching of size at least 5 . One can check that $H$ plus one additional edge in $A$ is already Hamiltonian-connected. Therefore, $G_{c}$ is Hamiltonian-connected, a contradiction. This proves $f_{\alpha+1}=\alpha+1$.

We now claim that $f_{\alpha}=\alpha+1$. Suppose not. Then $f_{\alpha}=\alpha$. As $f_{\alpha+1}=\alpha+1$, it follows that $B=\left\{u_{\alpha+1}, \ldots, u_{2 \alpha+1}\right\}$. So $|A|=\alpha$ and $|B|=\alpha+1$. Since $d\left(u_{\alpha+1}\right)=\alpha+1$ and $B$ is a clique, $u_{\alpha+1}$ has only one neighbor in $A$, say $x$. If every vertex in $B \backslash\left\{u_{\alpha+1}\right\}$ has degree $2 \alpha$, then $d(x) \geq|B|=\alpha+1$, contradicting the fact that $x \in A$. Thus, there exist some vertices in $B \backslash\left\{u_{\alpha+1}\right\}$ of degree at most $2 \alpha-1$. Among all such vertices, choose $u \in B \backslash\left\{u_{\alpha+1}\right\}$ such that $d(u)=2 \alpha-i$ is maximum. By Fact 4 , we have $2 \leq i \leq \alpha-1$. Suppose that $2 \leq i \leq \alpha-2$. By the similar argument as in Fact 5, there exists $A^{\prime}=A \backslash N(u)$ with $\left|A^{\prime}\right|=i$ such that $d(x) \leq i+1$ for any $x \in A^{\prime}$; and there also exists $B^{\prime} \subseteq B$ with $\left|B^{\prime}\right|=\alpha-i$ such that $d(y) \leq 2 \alpha-i$ for any $y \in B^{\prime}$ (except the vertex $u_{\alpha+1}$ ). Notice that in this case, 
$\left|B^{\prime}\right|=\alpha-i \geq 2$. So $e \leq \frac{1}{2}(i(i+1)+(\alpha-i) \alpha+(\alpha+1)+(\alpha-i-1)(2 \alpha-i)+(i+1)(2 \alpha))=$ $i^{2}-(\alpha-1) i+\frac{3 \alpha^{2}+\alpha+1}{2} \leq \frac{3 \alpha^{2}}{2}-\frac{\alpha}{2}+5<e$, where the second inequality holds as the maximum of $i^{2}-(\alpha-1) i$ occurs at either $i=2$ or $i=\alpha-2$. This shows that for any $u \in B \backslash\left\{u_{\alpha+1}\right\}, d(u)=\alpha+1$ or $2 \alpha$. If there are two vertices in $B \backslash\left\{u_{\alpha+1}\right\}$ of degree $\alpha+1$, then $e \leq \frac{1}{2}\left(\alpha^{2}+3(\alpha+1)+(\alpha-2)(2 \alpha)\right)=\frac{1}{2}\left(3 \alpha^{2}-\alpha+3\right)<e$, a contradiction. Hence, there exists only one vertex $u \in B \backslash\left\{u_{\alpha+1}\right\}$ with $d(u)=\alpha+1$. Then each of $\left\{u_{\alpha+1}, u\right\}$ has a neighbor in $A$, say $x, x^{\prime}$, respectively. We see that $x, x^{\prime}$ are distinct (as otherwise $x=x^{\prime}$ is adjacent to all vertices in $B$ and then $d(x) \geq \alpha+1)$. It is easy to see that $x, x^{\prime}$ have degree $\alpha$ in $H$, while all other vertices in $A$ have degree $\alpha-1$ in $H$. So $E(A)$ is a matching (if not empty). Since $e(H)=\left(\begin{array}{c}\alpha+1 \\ 2\end{array}\right)+2+(\alpha-1) \alpha=\frac{3 \alpha^{2}}{2}-\frac{\alpha}{2}+2$ and $e>\frac{3 \alpha^{2}}{2}-\frac{\alpha}{2}+5$, $E(A)$ is a matching of size at least 4 . We can verify that $H$ plus any edge in $A$ (which is independent of $x, x^{\prime}$ ) is Hamiltonian-connected; so $G_{c}$ is Hamiltonian-connected as well, a contradiction. This proves that $f_{\alpha}=\alpha+1$. Note that $|A|=\alpha-1$ and $|B|=\alpha+2$.

Lastly we claim that any vertex in $B \backslash\left\{u_{\alpha}, u_{\alpha+1}\right\}$ has degree $2 \alpha$ in $G_{c}$. Suppose this is not true. Then there exists a vertex $u \in B \backslash\left\{u_{\alpha}, u_{\alpha+1}\right\}$ with $d(u)=2 \alpha-i$, where $2 \leq i \leq$ $\alpha-1$, and subject to this, we choose $d(u)$ to be maximum. Similarly as above, there is a subset $A^{\prime}=A \backslash N(u)$ with $\left|A^{\prime}\right|=i$ such that each vertex in $A^{\prime}$ is of degree at most $i+1$. Take any $x \in A^{\prime}$. Then, there exists $B^{\prime} \subseteq B \backslash N(x)$ with $\left|B^{\prime}\right|=|B|-(i+1)=\alpha-i+1$ such that for any $y \in B^{\prime}, d(y) \leq 2 \alpha-i$. If $2 \leq i \leq \alpha-3$, then $\left|B^{\prime}\right|=\alpha-i+1 \geq 2$ and possibly $u_{\alpha}, u_{\alpha+1}$ are in $B^{\prime}$, thus we have $e \leq \frac{1}{2}(i(i+1)+(\alpha-1-i) \alpha+2(\alpha+1)+(\alpha-i-1)(2 \alpha-i)+(i+1)(2 \alpha))=$ $i^{2}-(\alpha-1) i+\frac{3 \alpha^{2}+\alpha+2}{2} \leq \frac{3 \alpha^{2}}{2}-\frac{\alpha}{2}+5<e$, a contradiction. If $i=\alpha-2$, then $d(u)=\alpha+2$, so $e \leq \frac{1}{2}(i(i+1)+(\alpha-1-i) \alpha+2(\alpha+1)+(\alpha+2)+(\alpha-1)(2 \alpha))=\frac{3 \alpha^{2}}{2}-\frac{\alpha}{2}+3<e$, also a contradiction. Hence, $i$ must be $\alpha-1$. This implies that every vertex in $B \backslash\left\{u_{\alpha}, u_{\alpha+1}\right\}$ has degree $2 \alpha$ or $\alpha+1$. If there are at least two vertices in $B \backslash\left\{u_{\alpha}, u_{\alpha+1}\right\}$ of degree $\alpha+1$, then $e \leq \frac{1}{2}((\alpha-1) \alpha+4(\alpha+1)+(\alpha-2)(2 \alpha))=\frac{3 \alpha^{2}}{2}-\frac{\alpha}{2}+2<e$. So $B$ has exactly three vertices $\left(u_{\alpha}, u_{\alpha+1}\right.$ and say $\left.u\right)$ of degree $\alpha+1$, while other vertices in $B$ have degree $2 \alpha$. Note that $\left(A, B-\left\{u_{\alpha}, u_{\alpha+1}, u\right\}\right)$ forms a complete bipartite $K_{\alpha-1, \alpha-1}$. This, together with the fact that $B$ induces a clique, shows that $G_{c}$ is Hamiltonian-connected, finishing the proof of this claim.

Now we see that $B$ induces a clique $K_{\alpha+2}, u_{\alpha}$ and $u_{\alpha+1}$ have no neighbors in $A$, and $\left(A, B-\left\{u_{\alpha}, u_{\alpha+1}\right\}\right)$ is complete bipartite. So every vertex in $A$ has $\alpha$ neighbors in $B$, which in turn shows that $A$ is stable. We have proved that $G_{c}=W_{c,\left\lfloor\frac{c}{2}\right\rfloor, c}$. The proof of Lemma 4.2 is completed.

\subsection{Proof of Lemma 4.3: an estimate of the clique number}

Lemma 4.3. Let $G$ be a 2-connected graph on $n$ vertices and $C$ be a locally maximal cycle in $G$ of length $c \leq n-1$. Suppose that $e(G)>\max \left\{f(n, k+1, c), f\left(n,\left\lfloor\frac{c}{2}\right\rfloor-1, c\right)\right\}$. If $G[C]$ contains a subset $S$ of $s-1$ vertices of degree at most $s$ in $G[C]$ for some integer $2 \leq s \leq\left\lfloor\frac{c}{2}\right\rfloor-1$ such that $G[C]-S$ is a clique, then $2 \leq s \leq k$ and the clique number of $G[C]$ is at least $c-k+1$.

To prove this, we will need some ingredient in the proof of [10] by Fan, Lv and Wang. An important tool in [10] is an edge-switching technique, which we introduce as follows. Let $x y$ be an edge in a graph $G$ and let $A \subset N(y) \backslash(N(x) \cup\{x\})$. The edge-switching graph of $G$ with respect to $A$ (from $y$ to $x$ ), denoted by $G[y \rightarrow x ; A]$, is the graph obtained from $G$ by deleting all the edges $y z, z \in A$ and adding all the edges $x z, z \in A$. 
Lemma 4.5 (Lemma 2.4, [10]). Let $G$ be a 2-connected graph, $C$ a locally maximal cycle in $G$, and $R$ a component in $G-C$. Then one of the following holds:

(i) $N_{R}(x)=V(R)$ for every vertex $x \in N_{C}(R)$;

(ii) There exists a vertex $y \in N_{R}(x)$ for some $x \in N_{C}(R)$ and a nonempty set $A \subseteq$ $N_{R}(y) \backslash\left(N_{R}(x) \cup\{x\}\right)$ such that

$$
G^{\prime}= \begin{cases}G[y \rightarrow x ; A] & \text { if } G[y \rightarrow x ; A] \text { is 2-connected }, \\ G[y \rightarrow x ; A]+y x^{\prime} & \text { otherwise. }\end{cases}
$$

is 2-connected, where $x^{\prime} \in N_{C}(R) \backslash\{x\}$, and $C$ remains a locally maximal cycle in $G^{\prime}$.

We now prove Lemma 4.3. We point out that the graph $G^{\prime}$ defined in Lemma 4.5)(ii) satisfies that $e\left(G^{\prime}\right) \geq e(G)$ and $G^{\prime}[C]=G[C]$.

Proof. When applying Lemma 4.5(ii), we see that the cycle $C$ remains locally maximal in the resulting graph, which is 2-connected. So we may repeatedly apply Lemma 4.5(ii). Note that as the set $A$ is nonempty, each time Lemma 4.5(ii) is applied, the number of edges not incident with $C$ strictly decreases. So this process will eventually stop (at some graph say $G^{*}$ ); and when it stops, (i) must occur for any component $R$ in $G^{*}-C$, i.e.,

every vertex $x \in N_{G^{*}}(R) \cap V(C)$ is adjacent to all vertices in $V(R)$ in $G^{*}$.

Let $\omega$ be the clique number of $G[C]=G^{*}[C]$. Then $\omega \geq|V(C) \backslash S| \geq c-s+1$. Also we have that $e(G) \leq e\left(G^{*}\right)$ and $e\left(G^{*}[C]\right)=e(G[C]) \leq\left(\begin{array}{c}c-s+1 \\ 2\end{array}\right)+(s-1) s$.

Let $R_{1}, R_{2}, \ldots, R_{t}$ be all components of $G^{*}-C$. For any $1 \leq i \leq t$, let $p_{i}=\mid N_{G^{*}}\left(R_{i}\right) \cap$ $V(C) \mid$, and $d_{i}$ be the length of the longest path between any two vertices in $N_{G^{*}}\left(R_{i}\right) \cap V(C)$ with all internal vertices in $R_{i}$. In view of (2), we see that $d_{i}-2$ denotes the length of the longest path in $R_{i}$. By a theorem of Erdös and Gallai (see [8, Theorem 2.6]), we have $e_{G^{*}}\left(R_{i}\right) \leq \frac{\left(d_{i}-2\right)\left|V\left(R_{i}\right)\right|}{2}$. Let $R_{\alpha}$ be the component in $G^{*}-C$ which attains the maximum of $\left\{d_{i}+2 p_{i}: 1 \leq i \leq t\right\}$, and let $p:=p_{\alpha}$ and $d:=d_{\alpha}$. Then

$$
\begin{aligned}
e(G) & \leq e\left(G^{*}\right) \leq e\left(G^{*}[C]\right)+\sum_{i}\left(\frac{\left(d_{i}-2\right)}{2}\left|V\left(R_{i}\right)\right|+p_{i} \cdot\left|V\left(R_{i}\right)\right|\right) \\
& \leq\left(\begin{array}{c}
c-s+1 \\
2
\end{array}\right)+(s-1) s+\frac{d+2 p-2}{2}(n-c) .
\end{aligned}
$$

Next we claim that $d+2 p \leq 2+2 s$. Suppose that $d+2 p \geq 3+2 s$. Consider the component $R:=R_{\alpha}$ in $G^{*}-C$. If $d=2$, then it follows $p \geq s+1$. Since $\left(N_{G^{*}}(R) \cap V(C)\right)^{+}$ is an independent set in $V(C)$ of size $p$ (otherwise, it would contradict that $C$ is locally maximal in $\left.G^{*}\right)$, we have $\omega \leq c-(p-1) \leq c-s$, a contradiction to that $\omega \geq c-s+1$. Now we may assume $d \geq 3$. This shows that $|V(R)| \geq 2$. Since $G^{*}$ is 2-connected, by (21), we see that $N_{G^{*}}(R) \cap V(C)$ is a strong attachment of $R$ to $C$. By Lemma 2.5)(i), $\omega \leq c-(d-1)(p-1)$. As $p \geq 2$, we have $\left(\frac{d-1}{2}-1\right)((p-1)-1) \geq 0$, which implies that $\frac{d-1}{2}(p-1) \geq \frac{d-1}{2}+(p-1)-1$, that is, $(d-1)(p-1) \geq d+2 p-5$. So $\omega \leq c-(d-1)(p-1) \leq$ $c-(d+2 p)+5 \leq c-2 s+2 \leq c-s$, again a contradiction. This proves the claim.

Combining the above bounds, we obtain that

$e(G) \leq\left(\begin{array}{c}c-s+1 \\ 2\end{array}\right)+(s-1) s+\frac{d+2 p-2}{2}(n-c) \leq\left(\begin{array}{c}c-s+1 \\ 2\end{array}\right)+s(n-c+s-1)=f(n, s, c)$.

If $k+1 \leq s \leq\left\lfloor\frac{c}{2}\right\rfloor-1$, then by the monotonicity of the function $f(n, k, c)$, it holds that $e(G) \leq \max \left\{f(n, k+1, c), f\left(n,\left\lfloor\frac{c}{2}\right\rfloor-1, c\right\}\right.$, a contradiction. Thus we must have $2 \leq s \leq k$ and then $\omega \geq c-s+1 \geq c-k+1$, finishing the proof of Lemma 4.3. 


\subsection{Proof of Lemma 4.4}

Lemma 4.4. Let $G$ be a 2-connected graph on $n$ vertices with $\delta(G) \geq k$ and $C$ be a locally maximal cycle in $G$ of length $c \leq n-1$. If the clique number of $G[C]$ is at least $c-k+1$, then $G \in\left\{W_{n, k, c}, Z_{n, k, c}\right\}$.

Proof. Consider any component $R$ in $G-C$. Let $T$ be a maximum strong attachment of $R$ to $C$ and $Q:=N_{C}(R) \backslash T$. Let $t:=|T|, q:=|Q|$ and $\omega$ be the clique number of $G[C]$. So $\omega \geq c-k+1$. We define the triple $\operatorname{ch}(R):=(t, q, \omega)$ to be the character of the component $R$; and we say a component $R$ is infeasible, if $\left|N_{C}(R)\right| \leq k-1$ and $\operatorname{ch}(R) \neq(2,0, c-k+1)$.

We now proceed by establishing a sequence of claims. An important step for our proof is to show that in fact there is no infeasible component $R$ in $G-C$.

Claim 1. For any component $R$ in $G-C$, both $\left(N_{C}(R)\right)^{+}$and $\left(N_{C}(R)\right)^{-}$are stable, and $\left|N_{C}(R)\right| \leq k$.

Proof. If $\left(N_{C}(R)\right)^{+}$contains an edge say $x^{+} y^{+}$, where $x, y \in N_{C}(R)$, then there exists an $(x, R, y)$-path $P$ and $C^{\prime}:=\left(C-\left\{x x^{+}, y y^{+}\right\}\right) \cup P \cup\left\{x^{+} y^{+}\right\}$is a longer cycle than $C$ with $\left|E\left(C^{\prime}\right) \cap E(C, G-C)\right|=2$, a contradiction. So $\left(N_{C}(R)\right)^{+}$and $\left(N_{C}(R)\right)^{-}$are stable. This implies that $c-k+1 \leq \omega \leq c-\left|N_{C}(R)\right|+1$, proving the claim.

Claim 2. For any infeasible component $R$ in $G-C$ with $\operatorname{ch}(R)=(t, q, \omega)$, we have $|V(R)| \geq 2$ and $t \geq 2$.

Proof. Suppose that $|V(R)|=1$, say $V(R)=\{x\}$. By Claim 1, we have $\left|N_{C}(x)\right| \leq k$. But $\delta(G) \geq k$. This shows that $\left|N_{C}(x)\right|=k$, a contradiction to the definition of an infeasible component. So $|V(R)| \geq 2$. As $G$ is 2-connected, we have at least two independent edges between $C$ and $R$, implying that $t \geq 2$.

Claim 3. For any infeasible component $R$ in $G-C, 2 \leq\left|N_{C}(R)\right| \leq k-2$.

Proof. Suppose not. Set $N:=N_{C}(R)$, then $|N|=k-1$. As $G$ is 2-connected, $|N| \geq 2$, implying that $k \geq 3$.

Suppose that $\omega \geq c-k+2$. Let $W$ be a maximum clique of size $\omega \geq c-k+2$ in $G[C]$ and $I:=V(C) \backslash W$. By Claim $1, N^{+}$is stable, so $\left|W \cap N^{+}\right| \leq 1$. By the inclusionexclusion principle, $c-k+2 \leq|W|=\left|W \cup N^{+}\right|+\left|W \cap N^{+}\right|-\left|N^{+}\right| \leq c+1-(k-1)$. This shows that $|W|=c-k+2,\left|W \cap N^{+}\right|=1$, and $W \cup N^{+}=V(C)$, the last of which implies that $I \subseteq N^{+}$. Similarly, we have $I \subseteq N^{-}$. Then $I^{+} \cup I^{-} \subseteq N$. So $k-1=|N| \geq\left|I^{+} \cup I^{-}\right|=\left|I^{+}\right|+\left|I^{-}\right|-\left|I^{+} \cap I^{-}\right|=2(k-2)-\left|I^{+} \cap I^{-}\right|$, implying that $\left|I^{+} \cap I^{-}\right| \geq k-3$. Let $C=x_{1} x_{2} \ldots x_{c} x_{1}$. Since $|I|=k-2$, it is not hard to see that $I=\left\{x_{i+1}, x_{i+3}, \ldots, x_{i+2 k-5}\right\}$ for some $i$. So $N=I^{+} \cup I^{-}=\left\{x_{i}, x_{i+2}, \ldots, x_{i+2 k-4}\right\}$. In this case, for any two $x_{j}, x_{j+2} \in N$, every $\left(x_{j}, R, x_{j+2}\right)$-path must be of length 2 , implying that $N_{R}\left(x_{j}\right)=N_{R}\left(x_{j+2}\right)=\{x\}$ for some $x \in V(R)$. So $x$ is the unique neighbor of $N_{C}(R)$ in $R$. Since $\delta(G) \geq k$ and $\left|N_{C}(R)\right|=k-1, x$ should have other neighbors in $R$ and thus $R-\{x\} \neq \emptyset$. But we also have $N_{C}(R-\{x\})=\{x\}$, contradicting that $G$ is 2-connected.

Now we may assume that $\omega=c-k+1$. Recall the definitions of $T, Q, t, q$, respectively. We have $t+q=\left|N_{C}(R)\right|=k-1$. Since $|V(R)| \geq 2$, the longest $(x, R, y)$-path for all $x, y \in T$ is of length at least 3. By Lemma 2.5(ii), we have $\omega \leq c-2(t-1)-q=$ $c-k-t+3$. If $t \geq 3$, then $\omega \leq c-k$, a contradiction. So $t=2$. If $q=0$, then $\operatorname{ch}(R)=(t, q, \omega)=(2,0, c-k+1)$, a contradiction. So we may assume that $t=2$ and 
$q \geq 1$. Let $T=\left\{x_{1}, x_{2}\right\}$ and $x_{1} y_{1}, x_{2} y_{2}$ be two independent edges in $E(R, C)$, where $y_{1}, y_{2} \in V(R)$. Suppose that $|V(R)|=2$. Then $V(R)=\left\{y_{1}, y_{2}\right\}$. Since $\delta(G) \geq k$, we have $d_{C}\left(y_{1}\right) \geq k-1$ and $d_{C}\left(y_{2}\right) \geq k-1$. So $N=N_{C}\left(y_{1}\right)=N_{C}\left(y_{2}\right)$ and every vertex in $N$ belongs to $T$. So $q=0$, a contradiction.

It remains to consider $|V(R)| \geq 3$. As $q \geq 1$, there exists some vertex $w \in Q$. By Lemma 2.4, we may assume that $y_{1}$ is the unique neighbor of $w$ in $R$. Then $y_{1}$ also is the unique neighbor of $x_{1}$ in $R$ (as otherwise counting $w, x_{1}, x_{2}$ in, we would have $t \geq 3$ ). Since $t=2$, the maximum matching between $(R, C)$ has size two, so by König's theorem [17], either $\left\{y_{1}, y_{2}\right\}$ or $\left\{y_{1}, x_{2}\right\}$ is a vertex cover in $(R, C)$. In the former case, let $z=y_{2}$ and $H=G[R]$; and in the latter case, let $z=x_{2}$ and $H=G\left[R \cup\left\{x_{2}\right\}\right]$. As $G$ is 2-connected and $|V(H)| \geq 3, H+y_{1} z$ is 2-connected; and every vertex in $H+y_{1} z$, except $y_{1}, z$, has the same degree as in $G$, which is at least $k$. Applying Theorem 2.2 to $H+y_{1} z$, there exists a $\left(y_{1}, z\right)$-path in $H+y_{1} z$ of length at least $k$. Clearly this path also lies in $H$, which implies an $\left(x_{1}, R, x_{2}\right)$-path of length at least $k+1$. By Lemma 2.5(ii) with $t=2$ and $d=k+1$, $\omega \leq c-k-q \leq c-k-1$, a contradiction. This proves Claim 3 .

Note that Claim 3 also shows that if there exist infeasible components in $G-C$, then $k \geq 4$.

Claim 4. For any infeasible component $R$ in $G-C,|V(R)| \geq 3$ and $R$ is not 2-connected.

Proof. If $|V(R)| \leq 2$, then by Claim 3, any vertex $u \in V(R)$ has degree at most $1+$ $\left|N_{C}(R)\right| \leq k-1$ in $G$, a contradiction. So $|V(R)| \geq 3$.

Suppose for a contradiction that $R$ is 2-connected. For any $x, y \in V(G)$, let $I_{x y}$ be 1 if $x y \in E(G)$ and 0 otherwise. Then for any $u \in V(R)$, we have $d_{R}(u)=d_{G}(u)-d_{C}(u) \geq$ $k-t-\sum_{v \in Q} I_{u v}$. By Theorem 2.2, for any two vertices $y, y^{\prime} \in V(R)$, there is a $\left(y, y^{\prime}\right)$-path of length at least

$$
\ell \geq \frac{\sum_{u \in V(R) \backslash\left\{y, y^{\prime}\right\}}\left(k-t-\sum_{v \in Q} I_{u v}\right)}{|V(R)|-2} \geq k-t-\frac{q}{|V(R)|-2} .
$$

First we consider that $|V(R)|=3$. In this case, $R$ is a triangle, say $V(R)=\left\{y_{1}, y_{2}, y_{3}\right\}$. For any $i$, it follows from $\left|N_{R}\left(y_{i}\right)\right|=2$ that $N_{C}\left(y_{i}\right) \geq k-2$. By Claim $3, N_{C}\left(y_{i}\right)=N_{C}(R)$ for each $i$ and thus $t=\left|N_{C}(R)\right|=k-2$ and $q=0$. By Lemma 2.5, $c-k+1 \leq \omega \leq c-3(k-3)$. So $k \leq 4$. Recall that $k \geq 4$. So we have $k=4, t=2, q=0$ and $\omega=c-3$. That is, $\operatorname{ch}(R)=(t, q, \omega)=(2,0, c-3)=(2,0, c-k+1)$, a contradiction.

Now we may assume that $|V(R)| \geq 4$. In this case, following the above inequality, we have $\ell \geq k-t-\frac{q}{2} \geq k-t-\frac{k-2-t}{2}=\frac{k-t}{2}+1$, where the last inequality holds as $t+q=\left|N_{C}(R)\right| \leq k-2$. By Lemma 2.5(i),

$$
c-k+1 \leq \omega \leq c-(\ell+1)(t-1) \leq c-\left(\frac{k-t}{2}+2\right)(t-1),
$$

which implies that $(k-t+4)(t-1) \leq 2(k-1)$, and thus

$$
k(t-3) \leq(t-4)(t-1)-2 .
$$

If $t \geq 4$, then $k \leq \frac{(t-4)(t-1)-2}{t-3}=(t-2)-\frac{4}{t-3} \leq k-2-q-\frac{4}{t-3} \leq k-2$, a contradiction. If $t=3$, this becomes that $0 \leq-4$, which is impossible. Thus $t=2$. Let $T=\left\{x_{1}, x_{2}\right\}$ and $x_{1} y_{1}, x_{2} y_{2}$ be two independent edges for $y_{1}, y_{2} \in V(R)$. Then any $v \in V(R) \backslash\left\{y_{1}, y_{2}\right\}$ 
has $N_{C}(v) \subseteq\left\{x_{1}, x_{2}\right\}$, so $d_{R}(v) \geq k-2$. Since $R$ is 2-connected, by Theorem 2.2, there is a ( $\left.y_{1}, y_{2}\right)$-path in $R$ of length at least $k-2$. By Lemma 2.5)(ii), $\omega \leq c-(k-1)-q \leq c-k$ if $q \geq 1$, a contradiction. So we have $q=0$ and $\omega=c-k+1$. In this case, we have $\operatorname{ch}(R)=(t, q, \omega)=(2,0, c-k+1)$. This proves this claim.

Claim 5. Let $R$ be an infeasible component in $G-C$ and $B$ an end-block of $R$ with the cut-vertex $b$. Let $T:=\left\{v \in V(C):\left|N_{B-b}(v)\right| \geq 2\right\}$ with $t:=|T|$. Then the following hold:

(i) $B$ is 2-connected with $|V(B)| \geq 5$;

(ii) For any $y \in V(B-b)$, there is a $(y, b)$-path in $B$ of length at least $\frac{2}{3}(k-t+1)$;

(iii) For any $y_{1}, y_{2} \in V(B-b)$, there is a $\left(y_{1}, y_{2}\right)$-path in $B$ of length at least $\frac{7}{12}(k-t)$;

(iv) $t \leq 2$.

Proof. Let $Q:=N_{C}(B-b) \backslash T$ and $q:=|Q|$. By Claim 3, we have $t+q \leq k-2$.

(i). For any $v \in V(B-b), d_{R}(v)=d_{G}(v)-d_{C}(v) \geq k-(k-2)=2$. So any end-block $B$ of $R$ is 2-connected and thus $|V(B)| \geq 3$. Suppose that $|V(B)| \in\{3,4\}$. First we claim that $\left|N_{C}(B-b)\right| \geq 2$. If $|V(B)|=3$, then it is clear, as $k \geq 4$ and every vertex in $B-b$ has degree at most 2 in $B$, there are at least 2 neighbors in $V(C)$. For $|V(B)|=4$, by the similar argument we also see that $\left|N_{C}(B-b)\right| \geq 2$, unless $k=4$ and $B$ is a $K_{4}$. In the latter case (say $N_{C}(B-b)=\{x\}$ and $k=4$ ), since $G$ is 2-connected, there exists some $x^{\prime} \in V(C) \backslash\{x\}$ which has a neighbor in $V(R-(B-b))$; as $B$ is a $K_{4}$, there exists an $\left(x, R, x^{\prime}\right)$-path of length at least 5 . By Lemma 2.5 (with $d=5$ and the strong attachment $\left\{x, x^{\prime}\right\}$ ), we have $c-3=\omega \leq c-4$, a contradiction. This proves that $\left|N_{C}(B-b)\right| \geq 2$. By Claim 4, there exists another end-block $B_{0}$ of $R$. Let $b_{0}$ be the cut-vertex of $R$ with $b_{0} \in V\left(B_{0}\right)$. As $\left|N_{C}(B-b)\right| \geq 2$, there exist $y \in V(B-b)$ and $y^{\prime} \in V\left(B_{0}-b_{0}\right)$ such that $y x, y^{\prime} x^{\prime} \in E(G)$ are independent edges, where $x, x^{\prime} \in V(C)$. As $B$ and $B_{0}$ are 2connected, there is a $\left(y, y^{\prime}\right)$-path of length at least 4. By Lemma 2.5 (with $d=6$ and the strong attachment $\left\{x, x^{\prime}\right\}$ ), we have $c-k+1 \leq \omega \leq c-5$, which implies that $k \geq 6$.

Suppose $|V(B)|=3$. Then obviously $B$ is a triangle, say $b y_{1} y_{2} b$. And $d_{C}\left(y_{i}\right) \geq k-2$ for $i=1,2$. On the other hand, $d_{C}\left(y_{i}\right) \leq\left|N_{C}(R)\right| \leq k-2$ for $i=1,2$. Thus, $y_{1}, y_{2}$ both are adjacent to all vertices in $N_{C}(B-b)$. So $T=N_{C}(B-b)$ and $t=|T|=k-2$. There is a $\left(y_{1}, y_{2}\right)$-path in $B$ of length 2. By Lemma 2.5 (with $d=4$ and the strong attachment $T)$, as $k \geq 6$, we obtain $\omega \leq c-3(k-3) \leq c-k$, a contradiction.

Suppose $|V(B)|=4$. Then $B$ contains a cycle of length 4 , say $b y_{1} y_{2} y_{3} b$. If $\left|N_{C}(B-b)\right| \leq$ $k-3$, then $d_{B}\left(y_{i}\right)=3$ for $i=1,2,3$, and this also implies that each of $y_{1}, y_{2}, y_{3}$ is adjacent to all vertices in $N_{C}(B-b)$. So $N_{C}(B-b)$ is a strong attachment of size $k-3$. Note that $B$ is a $K_{4}$. By Lemma 2.5 (with $d=5$ and the strong attachment $N_{C}(B-b)$ ), we have $\omega \leq c-4(k-4) \leq c-k$, where the last inequality holds as $k \geq 6$, a contradiction. So $\left|N_{C}(B-b)\right| \geq k-2$. By Claim $3, N_{C}(B-b)=N_{C}(R)$ is of size $k-2$. We claim that $N_{C}(B-b)$ is a strong attachment. If $Q \neq \emptyset$, choose $x \in Q$. Suppose that $y_{1}$ is the unique vertex in $N_{B-b}(x)$. Then by the degree condition, we see that $y_{2}$ and $y_{3}$ are adjacent to every other vertex in $B$ and have the same neighborhood $N_{C}(B-b)-\{x\}$ in $C$. So, $N_{C}(B-b)$ is also a strong attachment. If $Q=\emptyset$, then $N_{C}(B-b)=T$ is clearly a strong attachment. This proves the claim. By Lemma 2.5 (with $d=4$ and the strong attachment $N_{C}(B-b)$ ), we have $\omega \leq c-3(k-3) \leq c-k$ (since $k \geq 6$ ), a contradiction. This proves (i).

(ii). For $x, y \in V(G)$, let $I_{x y}=1$ if $x y \in E(G)$ and 0 otherwise. Then for any vertex $u \in V(B-b)$, we have $d_{B}(u) \geq k-t-\sum_{v \in Q} I_{u v}$. Since $B$ is 2-connected, by Theorem 
2.2. for any $y \in V(B-b)$ there is a $(y, b)$-path of length $\ell_{y b}$, such that

$$
\ell_{y b} \geq \frac{\sum_{u \in V(B-\{y, b\})} d_{B}(u)}{|B|-2} \geq k-t-\frac{q}{|B|-2} \geq \frac{2}{3}(k-t+1)
$$

where the last inequality holds because $|B| \geq 5$ and $q \leq k-t-2$. This proves (ii).

(iii). Recall that for any $u \in V(B-b), d_{B}(u) \geq k-t-\sum_{v \in Q} I_{u v}$. By Theorem 2.2. for any distinct $y, y^{\prime} \in V(B)$, there is a $\left(y, y^{\prime}\right)$-path of length $\ell_{y y^{\prime}}$ at least

$$
\begin{gathered}
\frac{\sum_{u \in V\left(B-\left\{y, y^{\prime}\right\}\right)} d_{B}(u)}{|B|-2}=\frac{\left(\sum_{u \in V\left(B-\left\{y, y^{\prime}, b\right\}\right)} d_{B}(u)\right)+d_{B}(b)}{|B|-2} \geq \\
\frac{\left(\sum_{u \in V\left(B-\left\{y, y^{\prime}, b\right\}\right)}\left(k-t-\sum_{v \in Q} I_{v u}\right)\right)+2}{|B|-2} \geq \frac{(|B|-3)(k-t)-q+2}{|B|-2} \geq k-t-\frac{k-t+q-2}{|B|-2} .
\end{gathered}
$$

On the other hand, $|B|^{2}-|B| \geq 2 e(B) \geq \sum_{u \in B-b}\left(k-t-\sum_{v \in Q} I_{v u}\right)+2=(|B|-1)(k-$ $t)-(q-2)$, which implies that $|B| \geq k-t-\frac{q-2}{|B|-1}$. So, $\frac{k-t-2}{|B|-2} \leq 1+\frac{q-2}{(|B|-1)(|B|-2)}$. Hence, $\frac{k-t+q-2}{|B|-2} \leq 1+\frac{q-2}{(|B|-1)(|B|-2)}+\frac{q}{|B|-2} \leq 1+\frac{q-2}{12}+\frac{q}{3}=1+\frac{5 q-2}{12} \leq \frac{5(k-t)}{12}$, since $|B| \geq 5$ and $q \leq k-t-2$. So $l_{y y^{\prime}} \geq k-t-\frac{k-t+q-2}{B \mid-2} \geq \frac{7(k-t)}{12}$. This proves (iii).

(iv). Suppose that $t \geq 3$. Since $T$ is a strong attachment, by (iii) and Lemma 2.5, we have that

$$
c-k+1 \leq \omega \leq c-\left(\frac{7(k-t)}{12}+1\right)(t-1),
$$

which implies that $(k-t)(7 t-19) \leq 0$. As $t \geq 3$, it follows $k \leq t$, a contradiction to $t \leq k-2-q \leq k-2$. This proves (iv).

Claim 6. There is no infeasible component in $G-C$. In other words, any component $R$ in $G-C$ has either $\left|N_{C}(R)\right|=k$ or $\operatorname{ch}(R)=(2,0, c-k+1)$.

Proof. Suppose that there exists an infeasible component $R$ in $G-C$. By Claim $4, R$ is not 2-connected, so there exist two end-blocks $B_{1}, B_{2}$ of $R$, with cut-vertices $b_{1}, b_{2}$, respectively. By Claim 5, each $B_{i}$ is 2-connected and for any vertex $y \in V\left(B_{i}-b_{i}\right)$, there exists a $\left(y, b_{i}\right)$-path in $B_{i}$ of length $\ell_{y b_{i}} \geq \frac{2(k-t+1)}{3} \geq \frac{2(k-1)}{3}$.

Suppose there exist distinct vertices $x \in N_{C}\left(B_{1}-b_{1}\right)$ and $x^{\prime} \in N_{C}\left(B_{2}-b_{2}\right)$. Then there exist $y \in B_{1}-b_{1}$ and $y^{\prime} \in B_{2}-b_{2}$ such that $x y, x^{\prime} y^{\prime}$ are two independent edges. So $\left\{x, x^{\prime}\right\}$ is a strong attachment of $R$ to $C$; and moreover, there exists an $\left(x, R, x^{\prime}\right)$ path of length at least $\ell_{y b_{1}}+\ell_{y^{\prime} b_{2}}+2 \geq \frac{4(k-1)}{3}+2$. By Lemma 2.5. as $k \geq 4$, we have $c-k+1 \leq \omega \leq c-\left(\frac{4}{3}(k-1)+1\right) \leq c-k$, a contradiction.

Therefore, we may assume that $N_{C}\left(B_{1}-b_{1}\right)=N_{C}\left(B_{2}-b_{2}\right)=\{x\}$ for some vertex $x \in V(C)$. Let $y$ be a neighbor of $x$ in $B_{1}-b_{1}$. Note that $d_{B_{1}}(u) \geq k-1$ for any $u \in B_{1}-b_{1}$. By Theorem 2.2, there exists a $\left(y, b_{1}\right)$-path of length at least $k-1$. Since $G$ is 2-connected, there exists an edge $x^{\prime} y^{\prime} \in E(G)$ with $x^{\prime} \in V(C-x)$ and $y^{\prime} \in V(R)-\left(B_{1}-b_{1}\right) \cup\left(B_{2}-b_{2}\right)$. Clearly $\left\{x, x^{\prime}\right\}$ is a strong attachment of $R$ to $C$ and using the above $\left(y, b_{1}\right)$-path, one can easily find an $\left(x, R, x^{\prime}\right)$-path of length at least $k+1$. By Lemma 2.5, we have $c-k+1 \leq$ $\omega \leq c-k$, a contradiction. This proves Claim 6.

In the remaining, we let $C=x_{1} x_{2} \ldots x_{c} x_{1}$ and take the index of $x_{i}$ under modulo $c$. By Dirac's theorem, $c \geq \min \{n, 2 k\}$. We also have $c \leq n-1$. This shows that $c \geq 2 k$. 
Claim 7. Let $R$ be a component in $G-C$ with $\left|N_{C}(R)\right|=k$. Then, there exists $i \in[c]$ such that $I:=\left\{x_{i+1}, x_{i+3}, \ldots, x_{i+2 k-3}\right\}$ is a stable set, $V(C) \backslash I$ is a clique of size $c-k+1$, and $N_{C}(R)=\left\{x_{i}, x_{i+2}, \ldots, x_{i+2 k-2}\right\}$; moreover, $|V(R)|=1$.

Proof. Let $N:=N_{C}(R)$. Let $W$ be a maximum clique in $G[C]$ and $I:=V(C) \backslash W$. By Claim $1, N^{+}$is stable and thus $\left|W \cap N^{+}\right| \leq 1$. By the inclusion-exclusion principle, we have $c-k+1 \leq|W|=\left|W \cup N^{+}\right|+\left|W \cap N^{+}\right|-\left|N^{+}\right| \leq c+1-k$. This shows that $|W|=c-k+1,\left|W \cap N^{+}\right|=1$, and $W \cup N^{+}=V(C)$. In particular, we have $I \subseteq N^{+}$. Similarly, one can show that $I \subseteq N^{-}$. Thus, $I^{+} \cup I^{-} \subseteq N$. So $k=|N| \geq\left|I^{+} \cup I^{-}\right|=\left|I^{+}\right|+$ $\left|I^{-}\right|-\left|I^{+} \cap I^{-}\right|=2(k-1)-\left|I^{+} \cap I^{-}\right|$, implying that $\left|I^{+} \cap I^{-}\right| \geq k-2$. Since $|I|=k-1$ (and $c \geq 2 k$ ), it is not hard to see that the indices of the vertices in $I$ must form an arithmetic progression with difference two, say $I=\left\{x_{i+1}, x_{i+3}, \ldots, x_{i+2 k-3}\right\}$ for some $i \in[c]$. Also since $I^{+} \cup I^{-} \subseteq N$ and $|N|=k$, it follows that $N_{C}(R)=N=\left\{x_{i}, x_{i+2}, \ldots, x_{i+2 k-2}\right\}$.

For any $x_{j}, x_{j+2}$ in $N_{C}(R)$, since $C$ is locally maximal, there exists some vertex $y \in$ $V(R)$ such that $N_{R}\left(x_{j}\right)=N_{R}\left(x_{j+2}\right)=\{y\}$. This further implies that $y$ is the unique neighbor in $R$ for every vertex in $N_{C}(R)$. If $|V(R)| \geq 2$, then $y$ is a cut-vertex of $G$, contradicting the fact that $G$ is 2-connected. Thus $|V(R)|=1$. This proves the claim.

Claim 8. Let $R$ be a component in $G-C$ with $\operatorname{ch}(R)=(2,0, c-k+1)$, and $T:=$ $N_{C}(R)$. Then there exists some integer $i \in[c]$ such that $T=\left\{x_{i}, x_{i+c-k}\right\}$ and $W=$ $G\left[\left\{x_{i}, x_{i+1}, \ldots, x_{i+c-k}\right\}\right]$ is a clique of size $c-k+1$; moreover, $G[R \cup T]$ is a clique of size $k+1$ and there are no edges between $V(W) \backslash T$ and $V(C) \backslash V(W)$.

Proof. Let $T=\{x, y\}$ and $W$ be a maximum clique in $G[C]$ of size $c-k+1$.

First we show that the longest $(x, y)$-path in $G[C]$ has length at least $c-k$, with equality if and only if $T=\left\{x_{i}, x_{i+c-k}\right\}$ for some integer $i \in[c], W=G\left[\left\{x_{i}, x_{i+1}, \ldots, x_{i+c-k}\right\}\right]$, and there are no edges between $V(W) \backslash T$ and $V(C) \backslash V(W)$. We first observe that there are two disjoint subpaths of $C$, say $L_{1}, L_{2}$ from $x, y$ to $V(W)$, respectively. Let the other end of $L_{i}$ be $a_{i}$. Then, as $W$ is a clique, there exists an $\left(a_{1}, a_{2}\right)$-path in $W$ through all vertices of $W$, which, together with $L_{1}$ and $L_{2}$, gives an $(x, y)$-path in $G[C]$ passing through all vertices of $W$. Since $|V(W)|=c-k+1$, this $(x, y)$-path has length at least $c-k$. Now suppose that the longest $(x, y)$-path has length exactly $c-k$. Let $P_{1}, P_{2}$ be the two $(x, y)$-subpaths on $C$. If $W$ intersects both $P_{1}-\{x, y\}$ and $P_{2}-\{x, y\}$, then we could find an $(x, y)$-path through all vertices of $W$ and thus it has length at least $c-k+1$, a contradiction. So we may assume that $V(W) \subseteq V\left(P_{1}\right)$. This further shows that $V(W)=V\left(P_{1}\right)$. That is, there exists $i \in[c]$ such that $T=\left\{x_{i}, x_{i+c-k}\right\}$ and $W=G\left[\left\{x_{i}, x_{i+1}, \ldots, x_{i+c-k}\right\}\right]$. In this case, if there is some edge $u v$ with $u \in V(W) \backslash T$ and $v \in V(C) \backslash V(W)$, then one can easily find an $\left(x_{i}, x_{i+c-k}\right)$-path of length at least $c-k+1$, a contradiction.

Next we show that the longest $(x, y)$-path in $G[C]$ has length exactly $c-k$ and moreover, $G[R \cup T]$ is a clique of size $k+1$. To see this, we notice that since $G$ is 2-connected, $G[R \cup T]+x y$ is 2-connected and every vertex in $G[R \cup T]+x y$, except $x$ and $y$, has degree at least $k$. By Theorem 2.2, the longest $(x, y)$-path $P$ in $G[R \cup T]+x y$ has length at least $k$, with equality if and only if $G[R \cup T]+x y$ is the union of some cliques $K_{k+1}$ 's which pairwise share the same vertices $x$ and $y$. For our case, as the deletion of $\{x, y\}$ only results in one component $R$, the equality holds if and only if $G[R \cup T]+x y$ is a clique $K_{k+1}$. It is also clear that $P$ lies in $G[R \cup T]$. Let $P^{\prime}$ be the longest $(x, y)$-path in $G[C]$, which is of length at least $c-k$. Then $C^{\prime}:=P \cup P^{\prime}$ is a cycle of length at least $c$ with the property that $\left|E\left(C^{\prime}\right) \cap E(C, G-C)\right|=2$. If $C^{\prime}$ has length at least $c+1$, it 
will contradict that $C$ is locally maximal. So $C^{\prime}$ must have length $c$, and thus the longest $(x, y)$-paths in $G[R \cup T]+x y$ and in $G[C]$ are of lengths exactly $k$ and $c-k$, respectively. This, together with the last paragraph, imply that $\{x, y\}=\left\{x_{i}, x_{i+c-k}\right\}$ for some $i \in[c]$, $W=G\left[\left\{x_{i}, x_{i+1}, \ldots, x_{i+c-k}\right\}\right]$ is a clique, and $G[R \cup T]+x y$ is a clique $K_{k+1}$. In particular, we see $x y \in E(G)$, so $G[R \cup T]$ is a clique $K_{k+1}$. This proves Claim 8 ,

Claim 9. If there exists a component $R$ in $G-C$ with $\operatorname{ch}(R)=(2,0, c-k+1)$, then $G=Z_{n, k, c}$.

Proof. Let $R$ be a component in $G-C$ with $\operatorname{ch}(R)=(2,0, c-k+1)$. By Claim 8 , we may assume that $T:=N_{C}(R)=\left\{x_{1}, x_{c-k+1}\right\}$ and $W=G\left[\left\{x_{1}, x_{2}, \ldots, x_{c-k+1}\right\}\right]$ is a clique.

Let $A:=V(C) \backslash V(W)$. We first show that for every $x \in A, N_{G}(x) \subseteq A \cup T$. Suppose not. In view of Claim 8 , we may assume that there exists another component $R^{\prime}$ in $G-C$ which has a neighbor $x$ in $A$ (because $x$ has no neighbors in $R$ or $W \backslash T$ ). By Claim 6, either $\left|N_{C}\left(R^{\prime}\right)\right|=k$ or $\operatorname{ch}\left(R^{\prime}\right)=(2,0, c-k-1)$. If $\left|N_{C}\left(R^{\prime}\right)\right|=k$, then by Claim $7 N_{C}\left(R^{\prime}\right)$ is a clique with vertices $\left\{x_{i}, x_{i+2}, \ldots, x_{i+2 k-2}\right\}$ for some $i \in[c]$. Since $x \in N_{C}\left(R^{\prime}\right) \cap A$ and $A$ only consists of $k-1$ consecutive vertices on $C$, there must be $y \in N_{C}\left(R^{\prime}\right) \cap(V(W) \backslash T)$. So $x y \in E(G)$, contradicting Claim 8 . So assume that $\operatorname{ch}\left(R^{\prime}\right)=(2,0, c-k-1)$. Then $N_{C}\left(R^{\prime}\right)=\left\{x_{j}, x_{j+(c-k)}\right\}$ for some $j \in[c]$, where $x_{j} \in A$. In this case, we also see that $x_{j} x_{j+(c-k)}$ is an edge between $V(W) \backslash T$ and $A$, a contradiction, finishing the proof.

Therefore, as $\delta(G) \geq k$ and $|A \cup T|=k+1$, we also see that $G[A \cup T]$ induces a $K_{k+1}$. Together with Claim 8, this shows that if $R$ is a component in $G-C$ with $\operatorname{ch}(R)=(2,0, c-k+1)$, then $G[C]$ is a union of a clique $K_{k+1}$ and another clique $K_{c-k+1}$ which share the vertices in $N_{C}(R)$.

Now consider any component $R_{0}$ in $G-C$ other than $R$. We just proved $N_{C}\left(R_{0}\right) \cap A=$ $\emptyset$, so $N_{C}\left(R_{0}\right) \subseteq V(W)$. By Claim [6, either $\left|N_{C}\left(R_{0}\right)\right|=k$ or $\operatorname{ch}\left(R_{0}\right)=(2,0, c-k-1)$. Assume that $\left|N_{C}\left(R_{0}\right)\right|=k$. Let $x_{i}, x_{i+2}, \ldots, x_{i+2 k-2}$ be the vertices of $N_{C}\left(R_{0}\right)$ for some $i$, which are in $V(W)$. Then there exist two vertices in $\left(N_{C}\left(R_{0}\right)\right)^{+}$, which are also in $V(W)$, a contradiction to Claim 1 that $\left(N_{C}\left(R_{0}\right)\right)^{+}$is stable. So we have $\operatorname{ch}\left(R_{0}\right)=(2,0, c-k-1)$. By the above paragraph, we must have $N_{C}\left(R_{0}\right)=N_{C}(R)$; moreover, by Claim $8, G\left[R_{0} \cup\right.$ $\left.N_{C}\left(R_{0}\right)\right]$ forms a clique $K_{k+1}$. This shows that $G=Z_{n, k, c}$, proving this claim.

Hence, by Claims 6, 7 and 9, we may assume that every vertex $y$ in $G-C$ is an isolated vertex with $\left|N_{C}(y)\right|=k$.

Claim 10. For any $y, y^{\prime} \in G-C$, it holds that $N_{C}(y)=N_{C}\left(y^{\prime}\right)$.

Proof. Suppose that $N_{C}(y) \neq N_{C}\left(y^{\prime}\right)$. Then there exist distinct indices $i, j \in[c]$ such that $N_{C}(y)=\left\{x_{i}, x_{i+2}, \ldots, x_{i+2 k-2}\right\}$ and $N_{C}\left(y^{\prime}\right)=\left\{x_{j}, x_{j+2}, \ldots, x_{j+2 k-2}\right\}$. Also $I:=$ $\left\{x_{i+1}, x_{i+3}, \ldots, x_{i+2 k-3}\right\}$ and $I^{\prime}:=\left\{x_{j+1}, x_{j+3}, \ldots, x_{j+2 k-3}\right\}$ are independent. Moreover, $W:=V(C) \backslash I$ and $W^{\prime}:=V(C) \backslash I^{\prime}$ are cliques. So $\left|I^{\prime} \cap W\right| \leq 1$, implying that $\left|I^{\prime} \cap I\right| \geq k-2$.

First consider the case that $c=2 k$. If $k=2$, then without loss of generality, we may assume that $N_{C}(y)=\left\{x_{1}, x_{3}\right\}, N_{C}\left(y^{\prime}\right)=\left\{x_{2}, x_{4}\right\}, I=\left\{x_{2}\right\}$, and $W=x_{1} x_{3} x_{4} x_{1}$ is a triangle. Then one can easily find a 5 -cycle $x_{2} y^{\prime} x_{4} x_{3} x_{1} x_{2}$, contradicting that $C$ is locally maximal. If $k \geq 3$, then $I^{\prime} \cap I \neq \emptyset$. This implies that the indies of the vertices in $I$ and in $I^{\prime}$ are of the same parity, so we must have $N_{C}(y)=N_{C}\left(y^{\prime}\right)$, a contradiction.

Hence we may assume that $c \geq 2 k+1$. In this case, as $N_{C}(y) \neq N_{C}\left(y^{\prime}\right)$, we see that $I \neq I^{\prime}$. Since $\left|I^{\prime} \cap I\right| \geq k-2$, it holds that $\left|I^{\prime} \cap I\right|=k-2$. If $k=2$, then $x_{j+1}$ is in the clique $W=V(C) \backslash\left\{x_{i+1}\right\}$. One of $x_{j-1}, x_{j+3}$ cannot be $x_{i+1}$ (by symmetry, say $x_{i+1} \neq x_{j+3}$ ). 
So $x_{j+1} x_{j+3} \in E(G)$. Then $\left(C-\left\{x_{j+1}, x_{j+2}\right\}\right) \cup x_{j} y^{\prime} \cup y^{\prime} x_{j+2} \cup x_{j+2} x_{j+1} \cup x_{j+1} x_{j+3}$ is a cycle which is longer than $C$, a contradiction. Now let $k \geq 3$. Then without loss of generality, we may assume that $j=i+2$. Since $x_{i+1}, x_{i+2 k} \in W^{\prime}$, we have $x_{i+2 k} x_{i+1} \in$ $E(G)$. Let $P$ be the unique subpath of $C$ from $x_{i+2}$ to $x_{i+2 k-2}$ which contains $x_{i+3}$, and let $P^{\prime}=x_{i+2 k} x_{i+1} \cup x_{i+1} x_{i} \cup x_{i} y \cup y x_{i+2} \cup P$ be a path from $x_{i+2 k}$ to $x_{i+2 k-2}$. Since $A:=\left(V(C) \backslash V\left(P^{\prime}\right)\right) \cup\left\{x_{i+2 k}, x_{i+2 k-2}\right\}=V(C)-\left\{x_{i}, x_{i+1}, \ldots, x_{i+2 k-3}\right\} \subseteq W$, there exists a path from $x_{i+2 k}$ to $x_{i+2 k-2}$ and consisting of the vertices in $A$, which, together with $P^{\prime}$, forms a cycle $C^{\prime}$ satisfying that $\left|C^{\prime}\right|>|C|$ and $\left|E\left(C^{\prime}\right) \cap E(C, G-C)\right| \leq 2$. This contradicts that $C$ is locally maximal, completing the proof of this claim.

We now prove that $G=W_{n, k, c}$. By Claim 10, we may assume that for all $y \in G-C$, $N_{C}(y)=\left\{x_{1}, x_{3}, \ldots, x_{2 k-1}\right\}$. By Claim $7, I=\left\{x_{2}, x_{4}, \ldots, x_{2 k-2}\right\}$ is an independent set and $W:=V(C) \backslash I$ is a clique of size $c-k+1$. Therefore, to prove $G=W_{n, k, c}$, it remains to show that for every vertex $x \in I, N_{G}(x)=\left\{x_{1}, x_{3}, \ldots, x_{2 k-1}\right\}$. Since $\delta(G) \geq k$, it suffices to show that any vertex $x_{i} \in I$ cannot be adjacent to some vertex $x$ in $V(C)-$ $\left\{x_{1}, x_{3}, \ldots, x_{2 k-1}\right\}$. Suppose for a contradiction that $x_{i} x \in E(G)$, where $i \in\{2,4, \ldots, 2 k-2\}$. As $I$ is independent, such $x$ must be in $V(C)-\left\{x_{1}, x_{2}, \ldots, x_{2 k-1}\right\}$. Let $P, P^{\prime}$ be two disjoint subpaths in the segment $x_{1} x_{2} \ldots x_{2 k-1}$ of $C$ from $x_{i}, x_{i+1}$ to $x_{1}, x_{2 k-1}$, respectively. Then $Q=x x_{i} \cup P \cup x_{1} y \cup y x_{i+1} \cup P^{\prime}$ is a path from $x$ to $x_{2 k-1}$ and passing through some vertex $y \in G-C$. Note that $A:=(V(C) \backslash V(Q)) \cup\left\{x, x_{2 k-1}\right\}=V(C)-\left\{x_{1}, x_{2}, \ldots, x_{2 k-2}\right\}$ is a subset of the clique $W$. So there exists a path from $x$ to $x_{2 k-1}$ and consisting of all vertices in $A$. This path, together with $Q$, forms a cycle $C^{\prime}$, which is longer than $C$ and $\left|E\left(C^{\prime}\right) \cap E(C, G-C)\right| \leq 2$, a contradiction. The proof of Lemma 4.4 is completed.

We now have finished the proof of Theorem 4.1 (and thus Theorem 1.13).

\section{Proofs of Theorems 1.10 and 1.11}

Theorem 1.10. Let $G$ be a 2-connected graph on $n$ vertices with $\delta(G) \geq k$ and let $C$ be a longest cycle in $G$ of length $c \in[10, n-1]$. If $e(G)>\max \left\{f(n, k+1, c), f\left(n,\left\lfloor\frac{c}{2}\right\rfloor, c\right)\right\}$, then $\bar{G}=W_{n, k, c}$ or $Z_{n, k, c}$, where $\bar{G}$ denotes the $C$-closure of $G$.

Proof. We derive this from Theorem 1.9, By the discussion in Subsection 2.1, $f\left(n,\left\lfloor\frac{c}{2}\right\rfloor, c\right) \geq$ $f\left(n,\left\lfloor\frac{c}{2}\right\rfloor-1, c\right)$. So we have $e(G)>\max \left\{f(n, k+1, c), f\left(n,\left\lfloor\frac{c}{2}\right\rfloor-1, c\right)\right\}$. By Theorem 1.9, either $\bar{G}=W_{n, k, c}$ or $Z_{n, k, c}, G \subseteq W_{n,\left\lfloor\frac{c}{2}\right\rfloor, c}$, or $G$ is a subgraph of a member of $\mathcal{X}_{n, c} \cup \mathcal{Y}_{n, c}$ (only when $k=2$ and $c$ is odd). If $G \subseteq W_{n,\left\lfloor\frac{c}{2}\right\rfloor, c}$ or $G$ is a subgraph of a member of $\mathcal{X}_{n, c} \cup \mathcal{Y}_{n, c}$, then it is easy to see that $e(G) \leq f\left(n,\left\lfloor\frac{c}{2}\right\rfloor, c\right)$, a contradiction to that $e(G)>f\left(n,\left\lfloor\frac{c}{2}\right\rfloor, c\right)$. So it must be that $\bar{G}=W_{n, k, c}$ or $Z_{n, k, c}$.

The proof of Theorem 1.11 is more involved, as we are not guaranteed to be able to use Theorem 1.9, This is because

$$
\max \left\{f(n, k, c), f\left(n,\left\lfloor\frac{c}{2}\right\rfloor-1, c\right)\right\} \geq \max \left\{f(n, k+1, c), f\left(n,\left\lfloor\frac{c}{2}\right\rfloor-1, c\right)\right\}
$$

holds only when $\left\lfloor\frac{c}{2}\right\rfloor-1 \geq k+1$. In fact when $c \leq 2 k+3$, this inequality can be reversed.

Theorem 1.11. Let $G$ be a 2-connected graph on $n$ vertices with $\delta(G) \geq k$ and let $C$ be a longest cycle in $G$ of length $c \in[10, n-1]$. If $e(G)>\max \left\{f(n, k, c), f\left(n,\left\lfloor\frac{c}{2}\right\rfloor-1, c\right)\right\}$, then either $G \subseteq W_{n,\left\lfloor\frac{c}{2}\right\rfloor, c}$, or $k=2$, c is odd and $G$ is a subgraph of a member of $\mathcal{X}_{n, c} \cup \mathcal{Y}_{n, c}$. 
Proof. Since $e(G)>f\left(n,\left\lfloor\frac{c}{2}\right\rfloor-1, c\right)=\left(\left\lfloor\frac{c}{2}\right\rfloor-1\right)(n-c)+h\left(c+1,\left\lfloor\frac{c}{2}\right\rfloor-1\right)$, it holds that either $e(G-C)+e(G-C, C)>\left(\left\lfloor\frac{c}{2}\right\rfloor-1\right)(n-c)$ or $e(G[C])>h\left(c+1,\left\lfloor\frac{c}{2}\right\rfloor-1\right)$. If the former case occurs, then by Theorem 1.12, either $G \subseteq W_{n,\left\lfloor\frac{c}{2}\right\rfloor, c}$, or $c$ is odd and $G$ is a subgraph of a member of $\mathcal{X}_{n, c} \cup \mathcal{Y}_{n, c}$ (if this occurs, then $k=2$ ). So we may assume that $e(G[C])>h\left(c+1,\left\lfloor\frac{c}{2}\right\rfloor-1\right)$. It suffices to show the following

Claim. Let $G$ be a 2-connected graph on $n$ vertices with $\delta(G) \geq k$ and $C$ be a locally maximal cycle in $G$ of length $c \in[10, n-1]$. If $e(G)>\max \left\{f(n, k, c), f\left(n,\left\lfloor\frac{c}{2}\right\rfloor-1, c\right)\right\}$ and $e(G[C])>h\left(c+1,\left\lfloor\frac{c}{2}\right\rfloor-1\right)$, then $G \subseteq W_{n,\left\lfloor\frac{c}{2}\right\rfloor, c}$.

The remaining proof is similar to the one of Theorem 4.1. Let $\bar{G}$ be the $C$-closure of $G$. By Lemma 2.7, $C$ remains a locally maximal cycle in $\bar{G}$; and by Lemma 2.8, $\bar{G}[C]$ is non-Hamiltonian-connected. Using Lemma 2.11, we see that one of the following holds:

(i) $\bar{G}[C]$ contains a subset of $\left\lfloor\frac{c}{2}\right\rfloor-1$ vertices of degree at most $\left\lfloor\frac{c}{2}\right\rfloor$ in $\bar{G}[C]$, or

(ii) $\bar{G}[C]$ contains a subset $S$ of $s-1$ vertices of degree at most $s$ in $\bar{G}[C]$ for some $2 \leq s \leq\left\lfloor\frac{c}{2}\right\rfloor-2$ such that $\bar{G}[C]-S$ is a clique.

Suppose that (i) holds. Lemma 4.2 implies $\bar{G}[C]=W_{c,\left\lfloor\frac{c}{2}\right\rfloor, c}$. Following the same augments in Theorem 4.1, we have $G \subseteq \bar{G} \subseteq W_{n,\left\lfloor\frac{2}{2}\right\rfloor, c}$. Now assume that (ii) holds. Since $e(\bar{G}) \geq$ $e(G)>\max \left\{f(n, k, c), f\left(n,\left\lfloor\frac{c}{2}\right\rfloor-1, c\right)\right\}$, by using $k$ instead of $k+1$ in Lemma 4.3, we derive that the clique number of $\bar{G}[C]$ is at least $c-k+2$. By Lemma 4.4, we have $\bar{G} \in\left\{W_{n, k, c}, Z_{n, k, c}\right\}$, but in each of the two graphs, the corresponding clique number is $c-k+1$, a contradiction. This proves the claim. Thus we have proved Theorem 1.11.

\section{Concluding remarks}

The approach used here seems to be applicable for the following problem of Füredi, Kostochka and Verstraëte in [15]: for $n \geq \frac{3 c}{2}$, to describe the structures of 2-connected $n$-vertex graphs with circumference at most $c$, where $c$ is even, and with at least $f\left(n, \frac{c}{2}-2, c\right)$ edges. We also wonder if a general and clear stability result can hold for $k$-connected graphs $G$ (or even for 3-connected graphs with minimum-degree at least $k$ ) for which $G$ has $n$ vertices, circumference $c$ and $e(G)>\max \left\{f(n, k+a, c), f\left(n,\left\lfloor\frac{c}{2}\right\rfloor-b, c\right)\right\}$ for fixed integers $a, b \geq 1$. Finally we would like to mention that some related problems can be found in [13].

Acknowledgement. The first author would like to thank Alexandr V. Kostochka for helpful discussions.

\section{References}

[1] B. Bollobás, Extremal Graph Theory, Academic Press, New York (1978).

[2] J. A. Bondy, Large cycles in graphs, Discrete Math. 1 1971/1972, no. 2, 121-132.

[3] J. A. Bondy, V. Chvátal, A method in graph theory, Discrete Math. 15 (1976), no. $2,111-135$.

[4] J. A. Bondy, U. S. R. Murty, Graph Theory, Graduate Texts in Mathematics, 244. Springer, New York, 2008. xii+651 pp. ISBN: 978-1-84628-969-9. 
[5] V. Chvátal, On Hamilton's ideals, J. Combin. Theory Ser. B 12 (1972), 163-168.

[6] G. A. Dirac, Some theorems on abstract graphs, Proc. London Math. Soc. (3-2) (1952), 69-81.

[7] P. Erdős, Remarks on a paper of Pósa, Magya Tud. Akad. Mat. Kutató Int. Közl. 7 (1962), 227-229.

[8] P. Erdős, T. Gallai, On maximal paths and circuits of graphs, Acta Math. Acad. Sci. Hungar. 10 (1959), 337-356.

[9] G. Fan, Long cycles and the codiameter of a graph I, J. Combin. Theory Ser. B 49 (1990), no. 2, 151-180.

[10] G. Fan, X. Lv, P. Wang, Cycles in 2-connected graphs, J. Combin. Theory Ser. B 92 (2004), no. 2, 379-394.

[11] R. J. Faudree, R. H. Schelp, Path Ramsey numbers in multiclorings, J. Combin. Theory Ser. B 19 (1975), 150-160.

[12] Z. Füredi, A. Kostochka, R. Luo, A stability version for a theorem of Erdös on nonhamiltonian graphs, Discrete Math. 340 (2017), 2688-2690.

[13] Z. Füredi, A. Kostochka, R. Luo, Extensions of a theorem of Erdős on nonhamiltonian graphs, J. Graph Theory 89 (2018), 176-193.

[14] Z. Füredi, A. Kostochka, R. Luo, J. Verstraëte, Stability in the Erdős-Gallai Theorem on cycles and paths, II, Discrete Math. 341 (2018), 1253-1263.

[15] Z. Füredi, A. Kostochka, J. Verstraëte, Stability in the Erdős-Gallai theorems on cycles and paths, J. Combin. Theory Ser. B 121 (2016), 197-228.

[16] Z. Füredi, M. Simonovits, The history of degenerate (bipartite) extremal graph problems, Bolyai Math. Studies 25 pp.169-264, Erdős Centennial (L. Lovász, I. Ruzsa, and V. T. Sós, Eds.) Springer, 2013. Also see arXiv:1306.5167

[17] D. König, Graphs and matrices, Mat. Fiz. Lapok 38 (1931), 116-119 (in Hugarian).

[18] G. N. Kopylov, Maximal paths and cycles in a graph, Dokl. Akad. Nauk SSSR 234(1977), 19-21. (English translation: Soviet Math. Dokl. 18 (1977), no. 3, 593596.)

[19] M. Lewin, On maximal circuits in directed graphs, J. Combin. Theory Ser. B 18 (1975), 175-179.

[20] B. Li, B. Ning, Spectral analogues of Erdős' and Moon-Moser's theorems on Hamilton cycles, Linear Multilinear Algebra 64 (2016), no. 11, 2252-2269.

[21] O. Ore, Arc coverings of graphs, Ann. Mat. Pura Appl. 55 (4) (1961) 315-321.

[22] Z. Ryjáček, On a closure concept in claw-free graphs, J. Combin. Theory Ser. B 70 (1997), no. 2, 217-224.

[23] D. R. Woodall, Maximal circuits of graphs I, Acta Math. Acad. Sci. Hungar. 28 (1976), 77-80. 